\title{
Discretely Conservative Finite-Difference Formulations for Nonlinear Conservation Laws in Split Form: Theory and Boundary Conditions
}

Travis C. Fisher, Mark H. Carpenter, Jan Nordström, Nail K. Yamaleev and Charles Swanson

\section{Linköping University Post Print}

N.B.: When citing this work, cite the original article.

Original Publication:

Travis C. Fisher, Mark H. Carpenter, Jan Nordström, Nail K. Yamaleev and Charles Swanson, Discretely Conservative Finite-Difference Formulations for Nonlinear Conservation Laws in Split Form: Theory and Boundary Conditions, 2012, Journal of Computational Physics.

http://dx.doi.org/10.1016/j.jcp.2012.09.026

Copyright: Elsevier

http://www.elsevier.com/

Postprint available at: Linköping University Electronic Press

http://urn.kb.se/resolve?urn=urn:nbn:se:liu:diva- 84553 


\section{Accepted Manuscript}

Discretely Conservative Finite-Difference Formulations for Nonlinear Conservation Laws in Split Form: Theory and Boundary Conditions

Travis C. Fisher, Mark H. Carpenter, Jan Nordström, Nail K. Yamaleev, Charles Swanson

PII: S0021-9991(12)00571-2

DOI: http://dx.doi.org/10.1016/j.jcp.2012.09.026

Reference: YJCPH 4234

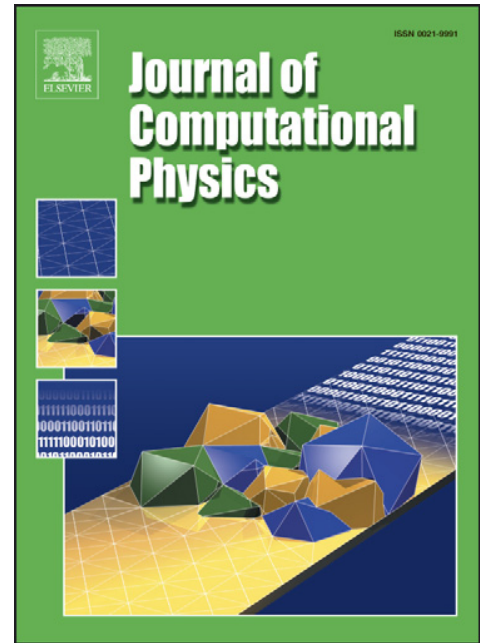

To appear in:

Journal of Computational Physics

Received Date:

17 October 2011

Revised Date: 25 July 2012

Accepted Date:

24 September 2012

Please cite this article as: T.C. Fisher, M.H. Carpenter, J. Nordström, N.K. Yamaleev, C. Swanson, Discretely Conservative Finite-Difference Formulations for Nonlinear Conservation Laws in Split Form: Theory and Boundary Conditions, Journal of Computational Physics (2012), doi: http://dx.doi.org/10.1016/j.jcp.2012.09.026

This is a PDF file of an unedited manuscript that has been accepted for publication. As a service to our customers we are providing this early version of the manuscript. The manuscript will undergo copyediting, typesetting, and review of the resulting proof before it is published in its final form. Please note that during the production process errors may be discovered which could affect the content, and all legal disclaimers that apply to the journal pertain. 


\title{
Discretely Conservative Finite-Difference Formulations for Nonlinear Conservation Laws in Split Form: Theory and Boundary Conditions
}

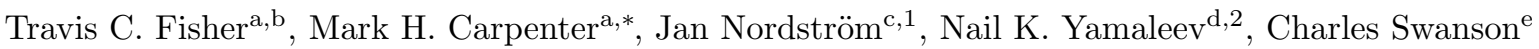 \\ ${ }^{a}$ Computational Aerosciences Branch, NASA Langley Research Center, Hampton, VA 23681, USA \\ ${ }^{b}$ School of Mechanical Engineering, Purdue University, West Lafayette, IN 47907, USA \\ ${ }^{c}$ Department of Mathematics, Linköping University, SE-581 83 Linköping, Sweden \\ ${ }^{d}$ Department of Mathematics, North Carolina A6T State University, Greensboro, NC 27411, USA \\ ${ }^{e}$ Distinguished Research Associate, NASA Langley Research Center
}

\begin{abstract}
The Lax-Wendroff theorem stipulates that a discretely conservative operator is necessary to accurately capture discontinuities. The discrete operator, however, need not be derived from the divergence form of the continuous equations. Indeed, conservation law equations that are split into linear combinations of the divergence and product rule form and then discretized using any diagonal-norm skew-symmetric summationby-parts (SBP) spatial operator, yield discrete operators that are conservative. Furthermore, split-form, discretely conservation operators can be derived for periodic or finite-domain SBP spatial operators of any order. Examples are presented of a fourth-order, SBP finite-difference operator with second-order boundary closures. Sixth- and eighth-order constructions are derived, and are supplied in an accompanying text file.
\end{abstract}

Keywords: high-order finite-difference methods, Lax-Wendroff, conservation, skew-symmetric, numerical stability.

\section{Introduction}

Consider the incompressible Navier-Stokes equations. The nonlinear product in the convective terms can be manipulated using the chain rule into many forms: 1) conservative (a.k.a. divergence), $\frac{\partial}{\partial x_{j}}\left(u_{i} u_{j}\right) ; 2$ ) primitive, $\left.u_{j} \frac{\partial u_{i}}{\partial x_{j}} ; 3\right)$ skew-symmetric, $\frac{1}{2} \frac{\partial}{\partial x_{j}}\left(u_{i} u_{j}\right)+\frac{1}{2} u_{j} \frac{\partial u_{i}}{\partial x_{j}}$; and 4) rotational, $u_{j}\left(\frac{\partial u_{i}}{\partial x_{j}}-\frac{\partial u_{j}}{\partial x_{i}}\right)-\frac{\partial}{\partial x_{j}}\left(\frac{1}{2} u_{i} u_{j}\right)$; as well as others. Although they are equivalent for smooth flows at the continuous level, each form can exhibit profoundly different discrete conservation, accuracy, and nonlinear stability properties. Growing evidence suggests that certain splittings of nonlinear conservation laws (e.g. the momentum terms in the equations of incompressible or compressible flow), deliver enhanced discrete accuracy and robustness, and in special cases result in the preservation of a secondary discrete invariant of the equations. This makes the use of split forms an attractive alternative to conventional approaches based on discretizations of the

\footnotetext{
* Corresponding author: 7578642318

Email addresses: travis.fisher@nasa.gov (Travis C. Fisher), mark.h.carpenter@nasa.gov (Mark H. Carpenter), jan.nordstrom@liu.se (Jan Nordström), nkyamale@ncat.edu (Nail K. Yamaleev), rcswanson11@yahoo.com (Charles Swanson)

${ }^{1}$ Work performed at NASA Langley Research Center during summer visitor program under technical monitor Joseph Morrison.

${ }^{2}$ Supported in part by NASA under grant NNX09AV08A and the Army Research Laboratory under grant W911NF-06-R-006. Preprint submitted to Elsevier 
divergence form of the equations. Evidence that supports this assertion is available in the following references $[1,2,3,4,5,6,7,8,9,10,11,12,13,14,15,16,17]$. Note that while some of these references focus entirely on the incompressible equations, others advocate elaborate splittings of the compressible equations (e.g. references $[12,14])$ to achieve desirable discrete properties.

It is well known that simulations of conservation law equations using nonconservative discrete operators could yield unphysical results, that are often indistinguishable from those that are physically correct to all but the most discriminating eye. This basic concept was rigorously established in the famous theorem of Lax and Wendroff [18], which stipulates that the conservation law form (divergence form) of the governing equations must be discretized with a consistent, discretely conservative operator (denoted a telescoping operator), if the weak solution is to be recovered upon grid convergence. (See Hou and Le Floch [19] for an insightful discussion on thy nonconservative schemes converge to wrong solutions.)

It would appear at first glance that split-form discrete operators are not discretely conservative because they are not derived from the divergence form of the continuous equations. Thus, despite favorable properties of the split forms, it would appear that they are inappropriate for use in simulations that capture discontinuities (e.g. inviscid Burgers equation or supersonic and hypersonic fluid flows).

There is growing evidence, however, that some discrete split forms of conservation laws can be manipulated into an equivalent, consistent, and telescoping form, thus allowing the Lax-Wendroff theorem to be used to guarantee that convergent captured discontinuities are weak solutions of the governing equations. For example, Ducros et al. [9] demonstrated that the skew-symmetric form of the convective terms in the Euler equations are discretely telescoping (at least for periodic fourth- and sixth-order centered operators), while Jameson [20] used the canonical form of the Burgers equation and developed discretely telescoping second order operators that are capable of capturing shocks. Recently Pirozzoli [15, 16] showed that all periodic centered finite-difference operators can be expressed in a telescoping form (even on curvilinear grids) if an appropriate splitting of the equations is used.

Several open issues must be resolved before high-fidelity simulations based on discretizations of alternative split forms of the equations become routine. One critical requirement is the need for high-order boundary closures and boundary conditions that maintain the desirable properties of the interior operators. Another is the need for a systematic methodology to extend alternative split-form single-domain operators to multiple domains, thereby extending the generality of any alternative approach.

The principle contribution of this work is a proof that demonstrates that any linear combination of the divergence and product-rule forms of nonlinear conservation laws, if discretized using any diagonal norm, summation-by-parts (SBP) operator, can be cast as a telescoping operator consistent with the divergence form of the conservation law. As such, any of these combinations is suitable for simulation of the conservation law, even in the presence of shocks. Three sets of telescoping, alternative split-form finite-difference operators 
are developed herein to illustrate these points: (2-4-2), (3-6-3) and (4-8-4). ${ }^{3}$ The shock capturing capability of the (2-4-2) split-form operator is tested using a one-dimensional shock tube problem, and is shown to yield shock locations equivalent to those obtained using conventional approaches. ${ }^{4} \mathrm{~A}$ final contribution of this work is the recognition that all single-domain alternative operators satisfy all of the necessary conditions for extension to general, multiblock discretizations, provided that interface coupling terms that maintain stability, accuracy, and conservation are used[23].

Several disclaimers are in order before proceeding. Appropriately splitting the conservation law form of the continuous equations allows for a rich set of novel discrete operators, all of which are shown herein to be discretely conservative. Split form equations derived using thermodynamic relations (e.g. pressure or entropy rather than energy) are outside the scope of this work. Furthermore, we do not suggest that the symmetrized canonical form of the Euler equation can be achieved using linear combinations of the divergence and product-rule forms of the Euler equations. No such transformation (to the best of our knowledge) exists. Finally, this work focuses on nondissipative SBP operators applied to split forms of conservation law equations. Clearly, if capturing shocks is the objective, then dissipation operators are needed that compliment the nondissipative split form operators. Rudimentary artificial viscosity methods are employed in this work to illustrate behavior of different split convective forms. A companion paper introduces a method that addresses shock-capturing and entropy stability of high order SBP finite difference operators.

The layout of the paper is as follows. Section 2 defines conventional SBP operators using matrix nomenclature, and then introduces several new lemmas that describe the structural properties of SBP operators. The section concludes with a variant of the original Lax-Wendroff theorem that is based on finite-domain SBP operators. Examples of a complete fourth-order operator are presented in section 3, followed by a theorem that proves that all split-forms of conservations laws are discretely telescoping when SBP operators are used as discretization operators. Section 4 discusses how alternative operators can be extended to complex geometries. In section 5, several split-form operators are applied to the viscous Burgers equation. Numerical validation of accuracy of an alternative form is given in section 6 , with the use of both single and multiple domains. For brevity, (3-6-3) and (4-8-4) operators are presented in an accompanying text file.

\section{Summation-by-parts Operators}

Consider a system of conservation laws of the form

$$
\begin{aligned}
& \frac{\partial q}{\partial t}+\frac{\partial f(q)}{\partial x}=0, \quad t \geq 0, \quad 0 \leq x \leq 1 \\
& q(x, 0)=q_{0}(x), q(0, t)=b c_{0}(t), q(1, t)=b c_{1}(t)
\end{aligned}
$$

\footnotetext{
${ }^{3}$ The $(p-2 p-p)$ nomenclature denotes that boundary-interior-boundary stencils are $p$-, $2 p$-, $p$-order accurate, respectively. The resulting operators are $(p+1)$-order accurate for hyperbolic equations (Euler) and incompletely parabolic equations (NavierStokes) and ( $p+2$ )-order accurate for parabolic equations (viscous Burgers) [21, 22].

${ }^{4}$ Dissipation is applied using an artificial viscosity method for both approaches.
} 
where $q=q(x, t)$ is the continuous solution vector, $f=f(q)$ is a nonlinear flux vector, and the initial data $q_{0}(x)$ is a bounded piecewise continuous function in $L_{2}$.

\subsection{Matrix Properties of Summation-By-Parts Operators}

Discretize the physical domain by using $N$ equidistant points that are distributed over the interval $[0,1]$ as

$$
\mathbf{x}=\left(x_{1}, x_{2}, \ldots, x_{N-1}, x_{N}\right), \quad x_{i}=(i-1) \frac{1}{N-1}, \quad i=1,2, \ldots, N
$$

and define a general class of SBP derivative operators $\mathcal{D}_{s b p}$ that satisfy the following matrix properties:

$$
\begin{aligned}
\mathcal{D}_{s b p} & =\mathcal{P}^{-1} \mathcal{Q}, \quad \mathcal{Q}+\mathcal{Q}^{T}=\mathcal{B}=\operatorname{diag}(-1,0, \cdots, 0,1) \\
\mathcal{P} & =\operatorname{diag}\left(p_{(1,1)}, \cdots, p_{(N, N)}\right), \quad \boldsymbol{\zeta}^{T} \mathcal{P} \boldsymbol{\zeta}>0, \quad \boldsymbol{\zeta} \neq \mathbf{0}
\end{aligned}
$$

where $p_{(1,1)} \cdots p_{(N, N)}$ are the elements of the diagonal matrix $\mathcal{P}$ and $\boldsymbol{\zeta}$ is an arbitrary vector. Without loss of generality, assume that $\mathcal{Q}$ is a banded matrix of half-bandwidth $r$. Because the SBP operators defined in eq. 2.2 utilize a diagonal norm $\mathcal{P}$ they are a subset of the more general block-norm SBP form (see ref. [23] or ref. [24]).

All SBP derivative operators discretely mimic the continuous integration-by-parts property. Given a smooth test function $\boldsymbol{\psi}=\left(\psi\left(x_{1}\right), \psi\left(x_{2}\right), \cdots, \psi\left(x_{N}\right)\right)^{T}$ the following manipulation follows immediately from the matrix definitions given in eq. (2.2).

$$
\begin{aligned}
\int_{0}^{1} \psi(x) f_{x} d x=\left.\psi f\right|_{0} ^{1}-\int_{0}^{1} \psi_{x} f d x & \approx \psi^{T} \mathcal{P} \mathcal{D}_{s b p} \mathbf{f} \\
& \approx \psi_{N} f_{N}-\psi_{1} f_{1}-\left(\mathcal{D}_{s b p} \boldsymbol{\psi}\right)^{T} \mathcal{P} \mathbf{f}
\end{aligned}
$$

The $\mathcal{P}$-norm provides the discrete quadrature weights of the integration. Note that the action of the differentiation operator $\mathcal{D}_{s b p}$ is smoothly transferred over to the test function $\psi$ without modification.

\subsubsection{Substructure of the $\mathcal{Q}$ Matrix}

The (Appendix A) proves that the row sums of all $\mathcal{Q}$ matrices are zero, and the column sums are zero except for the first and last, i.e.

$$
\mathcal{Q} \mathbf{1}=\mathbf{0} \quad ; \quad \mathbf{1}^{T} \mathcal{Q}=(-1,0, \cdots, 0,1)^{T} \quad ; \quad \mathbf{1}=(1, \cdots, 1)^{T}, \quad \mathbf{0}=(0, \cdots, 0)^{T}
$$

The $\mathcal{Q}$ matrices also have a telescoping structural consistency property (not reported previously, to our knowledge). The following lemma is used herein to prove flux consistency of the $\alpha$-split form of the equations. 
Lemma 2.1.

$$
\begin{gathered}
\frac{1}{2}=\sum_{k=1}^{r(i)} \sum_{l=1}^{k} q_{(i+l-k, i+l)} \\
1 \leq i+l, i+l-k \leq N \quad 1 \leq i \leq N-1
\end{gathered}
$$

is satisfied for $1 \leq i \leq N$, and $q_{(i, j)}$ is matrix element $(i, j)$ in the $\mathcal{Q}$ matrix. The nomenclature $r(i)$ here denotes the matrix half-bandwidth to emphasize that the upper dimension of the matrix is never exceeded, (i.e. $i+r(i) \leq N \quad, \quad \forall i)$.

Proof. Induction is used to show that eq. (2.5) is always satisfied for SBP operators. Recall that the sum of each row in $\mathcal{Q}$ is identically zero, and that the first element satisfies $q_{(1,1)}=-1 / 2$. Thus, the sum of the off-diagonal terms in the first row of $\mathcal{Q}$ satisfies $\frac{1}{2}=\sum_{l=1}^{r(1)} q_{(1,1+l)}$, which proves that $(2.5)$ holds for $i=1$.

Next, by traversing the matrix diagonal assume that eq. (2.5) is satisfied up to row $i$ : i.e. $\frac{1}{2}=$ $\sum_{k=1}^{r(i)} \sum_{l=1}^{k} q_{(i+l-k, i+l)}$. Now express the double sum for row $i+1$ in terms of the $i$ th double sum as

$$
\begin{aligned}
\sum_{k=1}^{r(i+1)} \sum_{l=1}^{k} q_{(i+1+l-k, i+1+l)} & =\sum_{k=1}^{r(i)} \sum_{l=1}^{k} q_{(i+l-k, i+l)}+\sum_{l=1}^{r(i+1)} q_{(i+1, i+1+l)}-\sum_{l=1}^{r(i+1)} q_{(i+1-l, i+1)} \\
& =\frac{1}{2}+\sum_{l=1}^{r(i+1)} q_{(i+1, i+1+l)}-\sum_{l=1}^{r(i+1)} q_{(i+1-l, i+1)}
\end{aligned}
$$

Because the sum of each row in $\mathcal{Q}$ is identically zero and $\mathcal{Q}$ is skew-symmetric, the desired result follows immediately

$$
\frac{1}{2}=\sum_{k=1}^{r(i+1)} \sum_{l=1}^{k} q_{(i+1+l-k, i+1+l)}
$$

Thus, eq. (2.5) is valid for $1 \leq i \leq N$.

Table (1) is a cartoon that summarizes how the structural property of $\mathcal{Q}$ defined in eq. (2.5) traverses down the diagonal of the matrix. Specifically, let $B B, C C$ and $D D$ in the cartoon represent the upper half of column $i+1$, the right half of row $i+1$, and the upper right quadrant of the matrix centered at position $(i+1, i+1)$, respectively. Equation (2.5) written at row $i$ becomes $\left.\frac{1}{2}=\sum_{k=1}^{r(i)} \sum_{l=1}^{k} q_{(i+l-k, i+l)}\right)=\sum B B+\sum D D$. Similarly, row $i+1$ is $\frac{1}{2}=\sum C C+\sum D D$, which follows immediately because $\sum B B=\sum C C$ by consistency and skew-symmetry of $\mathcal{Q}$.

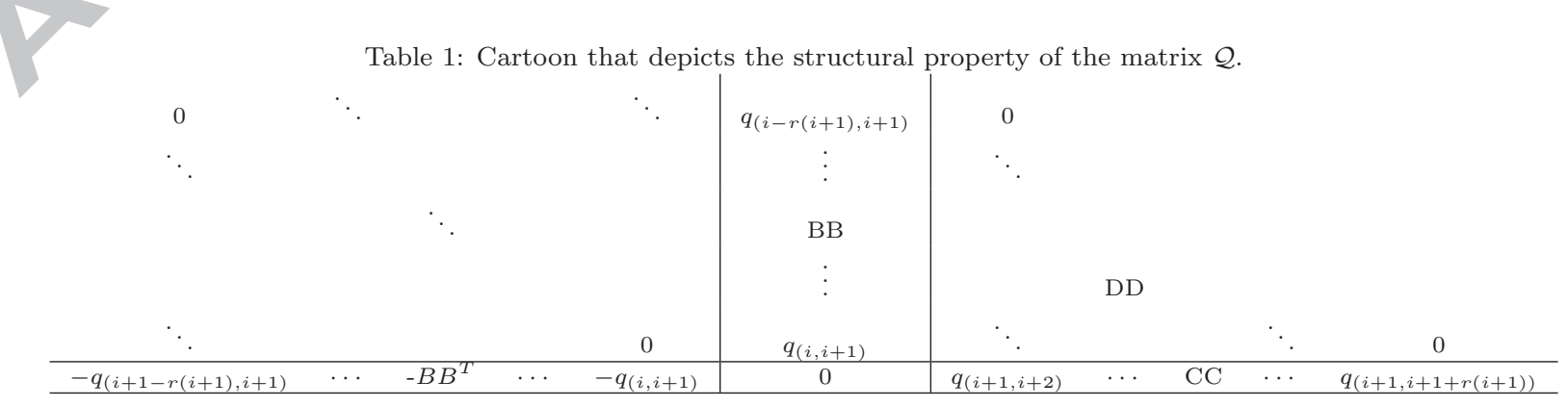




\subsubsection{An Elemental Decomposition of $\mathcal{Q}$}

An elemental decomposition exists for all $\mathcal{Q}$ matrices used in SBP operators. This decomposition is new (at least to our knowledge) and is instrumental in proving several theorems presented in the paper.

Define the $(N \times N)$ elemental difference matrices $\varepsilon^{i, i+l}, 1 \leq i<N-1,1 \leq l \leq r(i)$ as

$$
\varepsilon^{i, i+l}=\left[\begin{array}{ccc}
0 & 0 & 0 \\
0 & \tilde{\varepsilon}^{i, i+l} & 0 \\
0 & 0 & 0
\end{array}\right], \quad \tilde{\varepsilon}^{i, i+l}=\left[\begin{array}{ccccc}
-1 & 0 & \cdots & 0 & 1 \\
0 & 0 & \cdots & 0 & 0 \\
\vdots & \vdots & \vdots & \vdots & \vdots \\
0 & 0 & \cdots & 0 & 0 \\
-1 & 0 & \cdots & 0 & 1
\end{array}\right]
$$

where $\tilde{\varepsilon}^{i, i+l}$ is an $(l+1 \times l+1)$ difference matrix. The precise locations of the four nonzero elements of $\varepsilon^{i, i+l}$ are

$$
\varepsilon^{i, i+l}[[i, i]]=-1 ; \varepsilon^{i, i+l}[[i, i+l]]=+1 ; \varepsilon^{i, i+l}[[i+l, i]]=-1 ; \varepsilon^{i, i+l}[[i+l, i+l]]=+1
$$

Note that the $\tilde{\varepsilon}^{i, i+l}$ sub-matrix is positioned on the diagonal of $\varepsilon^{i, i+l} .^{5}$

Lemma 2.2. All $\mathcal{Q}$ matrices defined in eq. (2.2) support the following decomposition:

$$
\mathcal{Q}=\sum_{i=1}^{N-1} \sum_{l=1}^{r(i)} q_{i, i+l} \varepsilon^{i, i+l}
$$

where $q_{i, i+l}$ are the off-diagonal elements of $\mathcal{Q}$.

Proof. Consider the expansion

$$
\overline{\mathcal{Q}}=\sum_{i=1}^{N-1} \sum_{l=1}^{r(i)} q_{i, i+l} \varepsilon^{i, i+l}
$$

beginning first with the off-diagonal terms. The double sum $\sum_{i=1}^{N-1} \sum_{l=1}^{r(i)}$ of elemental matrices used in eq. (2.8), visits all sup-diagonal terms exactly once. Scaling each elemental contribution in the sum by $q_{i, i+l}$ simultaneously populates the sup- and sub-diagonal elements of $\overline{\mathcal{Q}}$ with precisely the off-diagonal values of the matrix $\mathcal{Q}$.

Moving next to the diagonal terms, note that each matrix $\tilde{\varepsilon}^{i, i+l}$ has two nonzero terms on the main diagonal, that are deposited on the main diagonal of $\overline{\mathcal{Q}}$. Summing all the diagonal contributions on each row of $\overline{\mathcal{Q}}$ yields

$$
\bar{q}_{i, i}=\sum_{l=1}^{r(i)}\left(q_{i, i+l}+q_{i, i-l}\right), \quad 1 \leq i \leq N
$$

Thus, the diagonal component $\bar{q}_{i, i}$ is the sum of all the off-diagonal terms in the corresponding row of $\mathcal{Q}$. The

\footnotetext{
${ }^{5}$ The bound $r(i)$ is the local half-bandwidth, and satisfies the relation $r(i) \leq r$. The use of the local bound $r(i)$ in the double sum, accounts for the contracting bandwidth in the corners of the $\mathcal{Q}$ matrix.
} 
sum of each row of $\mathcal{Q}$ is identically zero (See Appendix B1 for a proof of this statement), and the diagonal terms of $\mathcal{Q}$ satisfy the expression $\operatorname{diag}(\mathcal{Q})=[-1 / 2,0, \cdots, 0,1 / 2]$, which leads immediately to the the desired result

$$
\bar{q}_{i, i}=q_{i, i}, \quad 1 \leq i \leq N
$$

We state without proof the following lemma

Lemma 2.3. The transpose of all $\mathcal{Q}$ matrices defined in eq. (2.2) supports the following decomposition:

$$
\mathcal{Q}^{T}=\sum_{i=1}^{N-1} \sum_{l=1}^{r(i)} q_{i, i+l}\left[\varepsilon^{i, i+l}\right]^{T}
$$

Combining eq. (2.7) and lemma (2.3), and defining a new elemental operator $\delta^{i, i+l}$ as

$$
\left(\varepsilon^{i, i+l}-\left[\varepsilon^{i, i+l}\right]^{T}\right)=\delta^{i, i+l}
$$

yields the following relationship between elemental operators

$$
\mathcal{Q}-\mathcal{Q}^{T}=\sum_{i=1}^{N-1} \sum_{l=1}^{r(i)} q_{i, i+l}\left(\left[\varepsilon^{i, i+l}\right]-\left[\varepsilon^{i, i+l}\right]^{T}\right)=\sum_{i=1}^{N-1} \sum_{l=1}^{r(i)} q_{i, i+l} \quad\left(\delta^{i, i+l}\right)
$$

Note that the $(N \times N)$ elemental difference matrices $\delta^{i, i+l}, 1 \leq i<N-1,1 \leq l \leq r(i)$ have two nonzero elements located,

$$
\delta^{i, i+l}[[i, i+l]]=+2 ; \delta^{i, i+l}[[i+l, i]]=-2,
$$

and that elemental sub-matrices satisfy a relation similar to eq. (2.10), i.e.

$$
\left(\tilde{\varepsilon}^{i, i+l}-\left[\tilde{\varepsilon}^{i, i+l}\right]^{T}\right)=\tilde{\delta}^{i, i+l}
$$

\subsection{Complementary Grids}

All SBP operators approximating $f(q)_{x}$ can be implemented using a combination of two discrete matrix operators. The first is an interpolation step whereby flux data $f(q)$ is transferred to an intermediate location between each grid point. The second operator differentiates the fluxes back onto the original grid. As such, the two complementary sets of grid points (or meshes) are defined on the finite interval $0 \leq x \leq 1$, differ in dimension by one and are expressed by using the vectors

$$
\mathbf{x}=\left[x_{1}, x_{2}, \cdots, x_{N-1}, x_{N}\right]^{T}, \quad \overline{\mathbf{x}}=\left[\bar{x}_{0}, \bar{x}_{1}, \cdots, \bar{x}_{N-1}, \bar{x}_{N}\right]^{T}
$$


with $x_{1}=\bar{x}_{0}=0$ and $x_{N}=\bar{x}_{N}=1$. The points $\mathbf{x}$ are referred to as the "solution points," while the points $\overline{\mathbf{x}}$ are referred to as the "flux points." (The overbar nomenclature is reserved herein for those quantities that are defined at the flux points $\overline{\mathbf{x}}$.) The two point sets $\mathbf{x}$ and $\overline{\mathbf{x}}$ are interdigitated (except at boundaries, where they are collocated) and reflects the usual convention of introducing a flux point intermediate between two solution points. Further details on the definitions of the dual grids, as well as implementation details can be found elsewhere [25].

\subsection{Telescoping Derivative Operators}

Define the rectangular $(N \times N+1)$ difference matrix $\Delta$ (and for later use the related matrix $\tilde{\Delta})$

$$
\Delta=\left(\begin{array}{cccccc}
-1 & 1 & 0 & 0 & 0 & 0 \\
0 & -1 & 1 & 0 & 0 & 0 \\
0 & 0 & \ddots & \ddots & 0 & 0 \\
0 & 0 & 0 & -1 & 1 & 0 \\
0 & 0 & 0 & 0 & -1 & 1
\end{array}\right) \quad ; \quad \tilde{\Delta}=\left(\begin{array}{cccccc}
0 & 1 & 0 & 0 & 0 & 0 \\
0 & -1 & 1 & 0 & 0 & 0 \\
0 & 0 & \ddots & \ddots & 0 & 0 \\
0 & 0 & 0 & -1 & 1 & 0 \\
0 & 0 & 0 & 0 & -1 & 0
\end{array}\right)
$$

Definition 2.1. A telescoping derivative operator is any finite-domain operator $\mathcal{D}$ that can be expressed as

$$
f_{x} \approx \mathcal{D} \mathbf{f} \quad=\mathcal{P}^{-1} \Delta \mathcal{I} \mathbf{f}=\mathcal{P}^{-1} \Delta \overline{\mathbf{f}}
$$

where the flux vector $\overline{\mathbf{f}}=\mathcal{I} \mathbf{f}$ is constructed on $\overline{\mathbf{x}}$ using an appropriate interpolation operator $\mathcal{I}$. Furthermore, demand that the telescoping operator satisfies the endpoint flux consistency conditions

$$
\bar{f}_{0}=f\left(q_{1}\right) \quad, \quad \bar{f}_{N}=f\left(q_{N}\right)
$$

The matrix $\mathcal{P}^{-1}$ accounts for the appropriate grid spacings throughout the domain. The term telescoping reflects the fact that the $\Delta$ operator telescopes the flux from boundary to boundary.

Finally, define the boundary matrix $\Delta-\tilde{\Delta}=\tilde{\mathcal{B}}$, where $\tilde{\mathcal{B}}$ has two nonzero terms

$$
\tilde{\mathcal{B}}=\left(\begin{array}{cccccc}
-1 & 0 & 0 & 0 & 0 & 0 \\
0 & 0 & 0 & 0 & 0 & 0 \\
0 & 0 & \ddots & \ddots & 0 & 0 \\
0 & 0 & 0 & 0 & 0 & 0 \\
0 & 0 & 0 & 0 & 0 & 1
\end{array}\right)
$$

and accounts for the two terms that are telescoped out to the boundaries. Note that the matrix $\tilde{\Delta}$ can be 
thought of as being the negative of a difference matrix acting on a test function $\psi$, i.e.

$$
\boldsymbol{\psi}^{T} \tilde{\Delta}=\boldsymbol{\psi}_{x}^{T}+\mathcal{O}(d x)
$$

\subsubsection{Telescoping SBP Operators}

The following lemma proves that all SBP derivative operators $\mathcal{D}_{s b p}$ can be expressed in a telescoping form.

Define the $(N+1 \times N)$ elemental interpolation matrices $\gamma^{i, i+l}, 1 \leq i<N-1,1 \leq l \leq r(i)$ as

$$
\gamma^{i, i+l}=\left[\begin{array}{ccc}
0 & 0 & 0 \\
0 & \tilde{\gamma}^{i, i+l} & 0 \\
0 & 0 & 0
\end{array}\right], \quad \tilde{\gamma}^{i, i+l}=\left[\begin{array}{ccccc}
1 & 0 & \cdots & 0 & 1 \\
1 & 0 & \cdots & 0 & 1 \\
\vdots & \vdots & \vdots & \vdots & \vdots \\
1 & 0 & \cdots & 0 & 1 \\
1 & 0 & \cdots & 0 & 1
\end{array}\right]
$$

where $\tilde{\gamma}^{i, i+l}$ is an $(l \times l+1)$ interpolation matrix. The $\tilde{\gamma}^{i, i+l}$ matrix occupies the $[i+1, i] \rightarrow[i+l, i+l]$ sub-matrix in $\gamma^{i, i+l}$, positioned so that diagonals of $\gamma^{i, i+l}$ and $\tilde{\gamma}^{i, i+l}$ coincide. The precise locations of the $2 l$ nonzero elements of $\gamma^{i, i+l}$ are

$$
\gamma^{i, i+l}[[i+k, i]]=1 ; \gamma^{i, i+l}[[i+k, i+l]]=1 ; k=1,2, \ldots l
$$

We reiterate that bound $r(i)$ is the local half-bandwidth, and satisfies the relation $i+r(i) \leq N$.

Lemma 2.4. All differentiation matrices that satisfy the SBP convention given in eq. (2.2) are telescoping operators in the norm $\mathcal{P}$.

Proof. Expand $\mathcal{Q}^{T}$ into its elemental components as given in lemma (2.3). Using the elemental interpolants defined in eq. (2.17) straightforward matrix multiplication yields the following result

$$
\mathcal{Q}^{T}=\sum_{i=1}^{N-1} \sum_{l=1}^{r(i)} q_{i, i+l}\left[\varepsilon^{i, i+l}\right]^{T}=-\sum_{i=1}^{N-1} \sum_{l=1}^{r(i)} q_{i, i+l} \Delta\left[\gamma^{i, i+l}\right]=-\Delta \sum_{i=1}^{N-1} \sum_{l=1}^{r(i)} q_{i, i+l}\left[\gamma^{i, i+l}\right]
$$

Thus, it is clear that $\mathcal{Q}^{T}$ can be represented in telescoping form.

Next, note that $\mathcal{Q}$ and $\mathcal{Q}^{T}$ are related by the expression $\mathcal{Q}=\mathcal{B}-\mathcal{Q}^{T}$ (see eq. (2.2)). The boundary matrix $\mathcal{B}$ can also be represented in telescoping form as follows. Let $\overline{\mathcal{B}}$ be an $(N+1 \times N)$ boundary matrix 
defined as $\mathcal{B}=\Delta \overline{\mathcal{B}}$, with $\overline{\mathcal{B}}$ defined as

$$
\overline{\mathcal{B}}=\left(\begin{array}{cccccc}
1 & 0 & 0 & 0 & 0 & 0 \\
0 & 0 & 0 & 0 & 0 & 0 \\
0 & 0 & \ddots & \ddots & 0 & 0 \\
0 & 0 & 0 & 0 & 0 & 0 \\
0 & 0 & 0 & 0 & 0 & 1
\end{array}\right)
$$

Combining eqs. (2.2), (2.18), and (2.19) yields the desired result

$$
\mathcal{Q}=\Delta\left[\overline{\mathcal{B}}+\sum_{i=1}^{N-1} \sum_{l=1}^{r(i)} q_{i, i+l} \gamma^{i, i+l}\right]
$$

Remark: The interpolation matrix $\mathcal{I}$ appearing in definition (2.1) is precisely

$$
\mathcal{I}=\overline{\mathcal{B}}+\sum_{i=1}^{N-1} \sum_{l=1}^{r(i)} q_{i, i+l} \gamma^{i, i+l}
$$

\subsubsection{Generalized SBP Operators}

Equation (2.3) demonstrates that SBP operators defined in eq. (2.2) mimic the integration-by-parts property. Telescoping operators also mimic the integration-by-parts but in a different way.

Given any smooth test function $\boldsymbol{\psi}=\left(\psi\left(x_{1}\right), \psi\left(x_{2}\right), \cdots, \psi\left(x_{N}\right)\right)^{T}$ and definitions provided in eqs. (2.13) and (2.16), integrate $f(q)_{x}$ against $\psi$ using the $\mathcal{P}$-norm as quadrature weights to yield the following expression

$$
\begin{aligned}
\int_{0}^{1} \psi(x) f_{x} d x=\left.\psi f\right|_{0} ^{1}-\int_{0}^{1} \psi_{x} f d x & \approx \psi^{T} \mathcal{P} \mathcal{P}^{-1} \Delta \overline{\mathbf{f}} \\
& \approx \psi^{T} \tilde{\mathcal{B}} \overline{\mathbf{f}}+\boldsymbol{\psi}^{T} \tilde{\Delta} \overline{\mathbf{f}} \\
& \approx \psi_{N} f_{N}-\psi_{1} f_{1}-\psi_{x}{ }^{T} \overline{\mathbf{f}}+\mathcal{O}(d x)
\end{aligned}
$$

Note that the action of the telescoping differentiation operator $\mathcal{D}$ is smoothly transferred over to the test function $\psi$, but in so doing drops in order down to first. Equation (2.21) provides a weaker definition of telescoping conservation than the one given in the original definition of SBP operators (see eq. (2.3)).

\subsection{The Lax-Wendroff Theorem}

Although smooth solutions to eq. (2.1) do exist, no smoothness can be guaranteed due to the nonlinearity of $f(q)$. Thus, we seek a weak solution to eq. (2.1) such that the integral relation

$$
\int_{0}^{1} \psi\left(q_{t}+f(q)_{x}\right) d x=[\psi f(q)]_{0}^{1}+\int_{0}^{1}\left(\psi q_{t}-\psi_{x} f(q)\right) d x=0
$$


is satisfied for all smooth test functions $\psi(x, t)$. (Note that only the spatial derivative is manipulated in this derivation.) The original Lax-Wendroff theorem is proven for the fully discrete equations on an infinite domain. Herein we present a semi-discrete proof on a finite-domain.

Choose a vector-valued function $\bar{f}(q)$ that is both a function of $2 r$ compactly supported vector arguments and related to the flux $f(q)$ with the sole requirement that $\bar{f}(q, \cdots, q)=f(q)$. With these definitions, the Lax-Wendroff theorem [18] is as follows:

Theorem 2.5. Semi-discretize eq. (2.1) using a telescoping operator defined in eq. (2.1)to yield

$$
\mathbf{q}_{t}+\mathcal{P}^{-1} \Delta \overline{\mathbf{f}}=\mathcal{P}^{-1}\left(\mathbf{g}_{1}+\mathbf{g}_{\mathbf{N}}\right)
$$

where $\mathbf{g}_{\mathbf{1}}$ and $\mathbf{g}_{\mathbf{N}}$ contain boundary data that is imposed weakly in a stable and consistent fashion. If the discrete solution $\mathbf{q}$ converges boundedly almost everywhere to $\tilde{\mathbf{q}}$, then $\tilde{\mathbf{q}}$ is a weak solution to the continuous equation.

Proof. Multiply eq. (2.23) from the left by the integration quadrature weights $\mathcal{P}$ and by the smooth test function $\boldsymbol{\psi}=\left(\psi\left(x_{1}\right), \psi\left(x_{2}\right), \cdots, \psi\left(x_{N}\right)\right)^{T}$, then use eq. (2.15) to move the action of the flux derivative over to the test function:

$$
\begin{aligned}
\boldsymbol{\psi}^{T} \mathcal{P} \mathbf{q}_{t}+\boldsymbol{\psi}^{T} \Delta \overline{\mathbf{f}}-\boldsymbol{\psi}^{T} \mathbf{g}_{\mathbf{b}} & =\boldsymbol{\psi}^{T} \mathcal{P} \mathbf{q}_{t}+\boldsymbol{\psi} \tilde{\mathcal{B}} \overline{\mathbf{f}}+\boldsymbol{\psi} \tilde{\Delta} \overline{\mathbf{f}}-\boldsymbol{\psi}^{T} \mathbf{g}_{\mathbf{b}} \\
& =\psi_{N}\left(f_{N}+g_{N}\right)-\psi_{1}\left(f_{1}+g_{1}\right)+\boldsymbol{\psi}^{T} \mathcal{P} \mathbf{q}_{t}-\boldsymbol{\psi}_{x}{ }^{T} \overline{\mathbf{f}}+\mathcal{O}(d x)
\end{aligned}
$$

Because the penalties $\mathbf{g}_{\mathbf{1}}$ and $\mathbf{g}_{\mathbf{N}}$ go to zero as the mesh is refined, Equation (2.24) approaches eq. (2.22) in the limit as $d x \rightarrow 0$, which is the desired result.

\subsection{Split-Operator Consistency Requirements for Lax-Wendroff}

Consider the flux $f$ that is defined in eq. (2.1), which satisfies the property $f(q)=v(q) w(q)$. The general split flux which is composed of a linear combination of the divergence and the product-rule components, can be written as

$$
q_{t}+\alpha f(q)_{x}+(1-\alpha)\left[v(q) w(q)_{x}+w(q) v(q)_{x}\right]=0
$$

Note that while $v(q)$ or $w(q)$ could contain multiple terms (e.g. $v(q)=\rho U_{i}$ ), the splitting is still binary. A triple splitting (e.g. $f(q)=u(q) v(q) w(q))$ as was proposed by Kennedy and Gruber[13] is outside the scope of this work. Discretizing eq. (2.25) with an SBP spatial operator results in the following semidiscrete equation:

$$
\begin{gathered}
\mathbf{q}_{t}+\alpha \mathcal{P}^{-1} \mathcal{Q} W \mathbf{v}+(1-\alpha)\left(V \mathcal{P}^{-1} \mathcal{Q} \mathbf{w}+W \mathcal{P}^{-1} \mathcal{Q} \mathbf{v}\right)=0 \\
\mathbf{v}=\left[v\left(q_{1}\right), v\left(q_{2}\right), \ldots, v\left(q_{N}\right)\right]^{T}, \quad V=\operatorname{diag}(\mathbf{v}) \\
\mathbf{w}=\left[w\left(q_{1}\right), w\left(q_{2}\right), \ldots, w\left(q_{N}\right)\right]^{T}, \quad W=\operatorname{diag}(\mathbf{w})
\end{gathered}
$$


Equation 2.26 does not explicitly lead to a telescoping flux form $\mathcal{P}^{-1} \Delta \overline{\mathbf{f}}$. Thus, if the Lax-Wendroff theorem is to be used to guarantee convergence to the weak solution of the continuous equations, then the following conditions must be met:

1. The discrete spatial operator $\alpha \mathcal{P}^{-1} \mathcal{Q} W \mathbf{v}+(1-\alpha)\left(V \mathcal{P}^{-1} \mathcal{Q} \mathbf{w}+W \mathcal{P}^{-1} \mathcal{Q} \mathbf{v}\right)$ must be equivalent to a telescoping form $\mathcal{P}^{-1} \Delta \overline{\mathbf{f}}$.

2. Each term in the flux $\overline{\mathbf{f}}_{i}$ must satisfy $\bar{f}(q, \cdots, q)=f(q)$, where $f$ is the conservation law flux that satisfies the jump conditions (e.g. Rankine-Hugoniot relations) $S[u]_{x_{d}^{-}}^{x_{d}^{+}}-[f]_{x_{d}^{-}}^{x_{d}^{+}}=0$. (Here $x_{d}^{-}$and $x_{d}^{+}$ represent the left and right side of a discontinuity located at $x_{d}$, respectively.)

\section{A Discretely Conservative, Finite-Domain, Split Form}

\subsection{A Fourth-Order Example}

Consider the SBP differentiation operator $\mathcal{D}_{2-4-2}$ written in the form

$$
\mathcal{D}=\frac{1}{\delta x}\left(\begin{array}{ccccccccccc}
-\frac{24}{17} & \frac{59}{34} & -\frac{4}{17} & -\frac{3}{34} & 0 & 0 & 0 & 0 & 0 & 0 & 0 \\
-\frac{1}{2} & 0 & \frac{1}{2} & 0 & 0 & 0 & 0 & 0 & 0 & 0 & 0 \\
\frac{4}{43} & -\frac{59}{86} & 0 & \frac{59}{86} & -\frac{4}{43} & 0 & 0 & 0 & 0 & 0 & 0 \\
\frac{3}{98} & 0 & -\frac{59}{98} & 0 & \frac{32}{49} & -\frac{4}{49} & 0 & 0 & 0 & 0 & 0 \\
0 & 0 & \frac{1}{12} & -\frac{2}{3} & 0 & \frac{2}{3} & -\frac{1}{12} & 0 & 0 & 0 & 0 \\
0 & 0 & 0 & \ddots & \ddots & \ddots & \ddots & \ddots & 0 & 0 & 0 \\
0 & 0 & 0 & 0 & \frac{1}{12} & -\frac{2}{3} & 0 & \frac{2}{3} & -\frac{1}{12} & 0 & 0 \\
0 & 0 & 0 & 0 & 0 & \frac{4}{49} & -\frac{32}{49} & 0 & \frac{59}{98} & 0 & -\frac{3}{98} \\
0 & 0 & 0 & 0 & 0 & 0 & \frac{4}{43} & -\frac{59}{86} & 0 & \frac{59}{86} & -\frac{4}{43} \\
0 & 0 & 0 & 0 & 0 & 0 & 0 & 0 & -\frac{1}{2} & 0 & \frac{1}{2} \\
0 & 0 & 0 & 0 & 0 & 0 & 0 & \frac{3}{34} & \frac{4}{17} & -\frac{59}{34} & \frac{24}{17}
\end{array}\right)
$$

where the diagonal matrix $\mathcal{P}$ is

$$
\mathcal{P}=\delta x \operatorname{diag}\left(\begin{array}{lllllllllll}
\frac{17}{48} & \frac{59}{48} & \frac{43}{48} & \frac{49}{48} & 1 & \cdots & 1 & \frac{49}{48} & \frac{43}{48} & \frac{59}{48} & \frac{17}{48}
\end{array}\right)
$$

The skew-symmetric matrix $\mathcal{Q}$ follows immediately from $\mathcal{P} \mathcal{D}$.

We begin by demonstrating that the fourth-order finite-domain split-form operator is discretely telescoping for any value of the parameter $\alpha$. The discrete split operator that is defined in eq. 2.26 can be written in telescoping form by using a linear combination of the divergence and product-rule fluxes as

$$
\mathbf{q}_{t}+\mathcal{P}^{-1} \Delta \overline{\mathbf{f}}=0, \quad \overline{\mathbf{f}}=\alpha \overline{\mathbf{f}}_{c}+(1-\alpha) \overline{\mathbf{f}}_{e}
$$


The divergence form flux follows immediately and is given by

$$
\overline{\mathbf{f}}_{c}=\left(\begin{array}{c}
w_{1} v_{1} \\
\frac{1}{96}\left(48 w_{1} v_{1}+59 w_{2} v_{2}-8 w_{3} v_{3}-3 w_{4} v_{4}\right) \\
\frac{1}{96}\left(-11 w_{1} v_{1}+59 w_{2} v_{2}+51 w_{3} v_{3}-3 w_{4} v_{4}\right) \\
\frac{1}{96}\left(-3 w_{1} v_{1}+51 w_{3} v_{3}+56 w_{4} v_{4}-8 w_{5} v_{5}\right) \\
\frac{1}{12}\left(-w_{3} v_{3}+7 w_{4} v_{4}+7 w_{5} v_{5}-w_{6} v_{6}\right) \\
\vdots \\
\frac{1}{12}\left(-w_{i-1} v_{i-1}+7 w_{i} v_{i}+7 w_{i+1} v_{i+1}-w_{i+2} v_{i+2}\right) \\
\vdots \\
\frac{1}{12}\left(-w_{N-5} v_{N-5}+7 w_{N-4} v_{N-4}+7 w_{N-3} v_{N-3}-w_{N-2} v_{N-2}\right) \\
\frac{1}{96}\left(-8 u_{N-4} v_{N-4}+56 u_{N-3} v_{N-3}+51 u_{N-2} v_{N-2}-3 u_{N} v_{N}\right) \\
\frac{1}{96}\left(-3 w_{N-3} v_{N-3}+51 w_{N-2} v_{N-2}+59 w_{N-1} v_{N-1}-11 w_{N} v_{N}\right) \\
\frac{1}{96}\left(-3 w_{N-1} v_{N-1}-8 w_{N-2} v_{N-2}+59 w_{N-1} v_{N-1}+48 w_{N} v_{N}\right) \\
w_{N} v_{N}
\end{array}\right)
$$


while the product-rule flux is

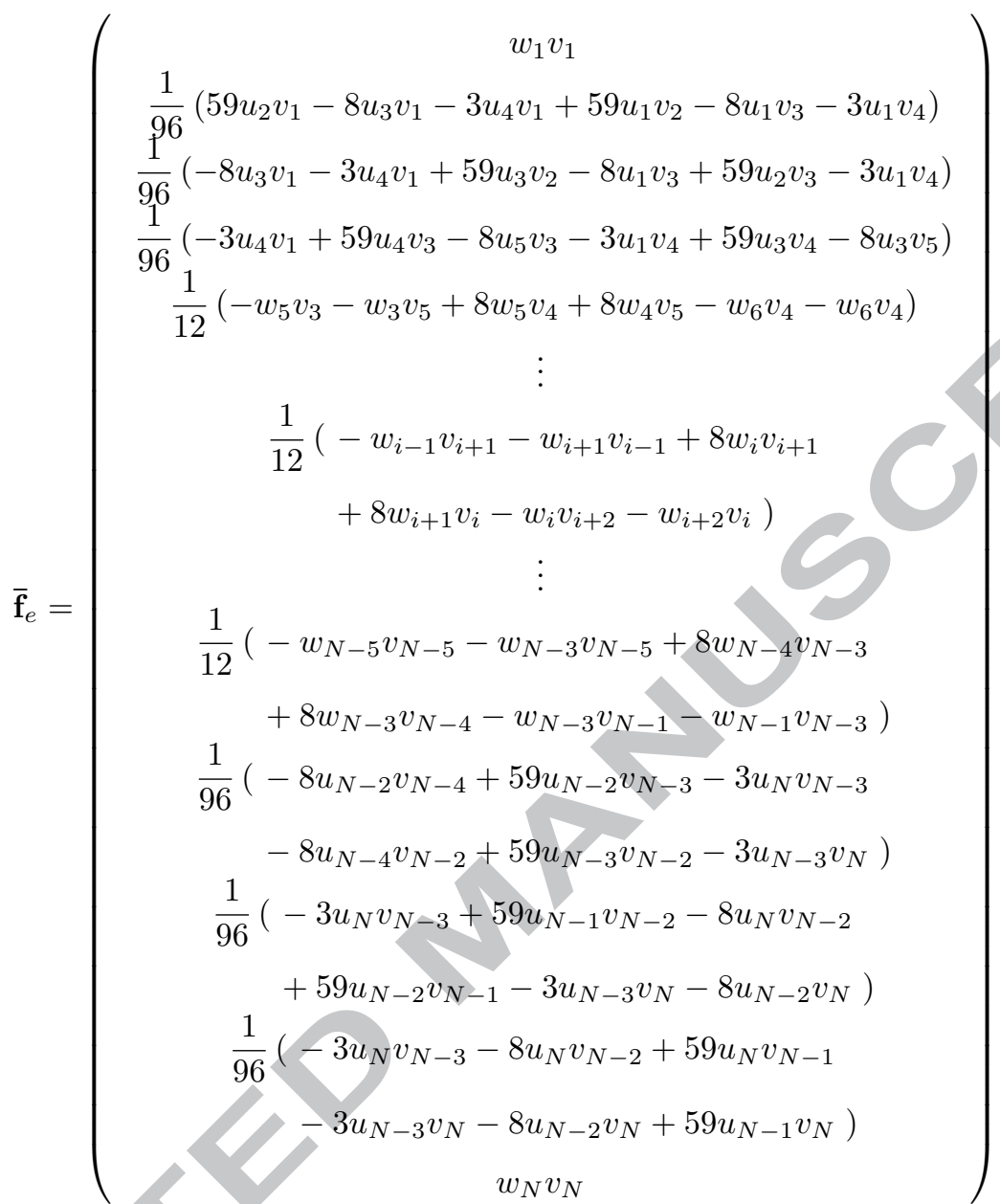

We also have constructed operators for $2 p=6$ and $2 p=8$ with boundary closure blocks of dimension $s=7$ and $s=9$, respectively. The interior flux forms for the sixth- and eight-order schemes are presented in Appendix C. The full flux form operators are available in an accompanying text file for brevity of this document and ease of implementation.

\subsection{All Diagonal-Norm SBP Operators}

The previous fourth-order finite-domain example (as well as the sixth- and eighth-order examples, which are given in Appendix C) suggests that conventional SBP operators lead to telescoping finite-domain splitform operators. This brings us to the two central theorems of this work.

Theorem 3.1. The discrete split-form operator (see eq. (2.26)) can be manipulated into the telescoping form

$$
\alpha \mathcal{P}^{-1} \mathcal{Q} W \mathbf{v}+(1-\alpha)\left(V \mathcal{P}^{-1} \mathcal{Q} \mathbf{w}+W \mathcal{P}^{-1} \mathcal{Q} \mathbf{v}\right)=\mathcal{P}^{-1} \Delta \overline{\mathbf{f}}
$$

for any diagonal-norm SBP operator that can be expressed in the form of eq. (2.2) and for any value of the 
parameter $\alpha$.

Proof. The term $\mathcal{P}^{-1} \mathcal{Q} W \mathbf{v}$ is already discretely telescoping as shown in lemma (2.4). Thus the split spatial operator is discretely telescoping for any value of the parameter $\alpha$ if

$$
\left(V \mathcal{P}^{-1} \mathcal{Q} \mathbf{w}+W \mathcal{P}^{-1} \mathcal{Q} \mathbf{v}\right)=\mathcal{P}^{-1} \Delta \overline{\mathbf{f}}_{e}
$$

for any general banded matrix $\mathcal{Q}$ of halfwidth $r$.

Begin by multiplying $\left(V \mathcal{P}^{-1} \mathcal{Q} \mathbf{w}+W \mathcal{P}^{-1} \mathcal{Q} \mathbf{v}\right)$ from the left by the diagonal matrix $\mathcal{P}$ to eliminate $\mathcal{P}^{-1}$ from the expression. Manipulate the resulting expression using $\frac{1}{2} \mathcal{Q}=\frac{1}{2}\left(\mathcal{B}-\mathcal{Q}^{T}\right)$ followed by the substitution of eq. (2.11), to yield

$$
\begin{aligned}
\mathcal{P}\left(V \mathcal{P}^{-1} \mathcal{Q} \mathbf{w}+W \mathcal{P}^{-1} \mathcal{Q} \mathbf{v}\right)= & ([V \mathcal{Q} \mathbf{w}+W \mathcal{Q} \mathbf{v}) \\
= & \frac{1}{2}\left([V \mathcal{B} \mathbf{w}+W \mathcal{B} \mathbf{v}]+V\left[\mathcal{Q}-\mathcal{Q}^{T}\right] \mathbf{w}+W\left[\mathcal{Q}-\mathcal{Q}^{T}\right] \mathbf{v}\right) \\
= & \frac{1}{2} \Delta \overline{\mathcal{B}}(V \mathbf{w}+W \mathbf{v}) \\
& +\frac{1}{2} \sum_{i=1}^{N-1} \sum_{l=1}^{r(i)} q_{i, i+l}\left[V\left(\varepsilon^{i, i+l}-\left[\varepsilon^{i, i+l}\right]^{T}\right) \mathbf{w}+W\left(\varepsilon^{i, i+l}-\left[\varepsilon^{i, i+l}\right]^{T}\right) \mathbf{v}\right] \\
= & \frac{1}{2} \Delta \overline{\mathcal{B}}(V \mathbf{w}+W \mathbf{v})+\frac{1}{2} \sum_{i=1}^{N-1} \sum_{l=1}^{r(i)} q_{i, i+l}\left[V \delta^{i, i+l} \mathbf{w}+W \delta^{i, i+l} \mathbf{v}\right]
\end{aligned}
$$

The task now is to express each term in the double sum as the product of the telescoping operator $\Delta$ and a flux. It is easy to verify that the double sum term, for all values of $i$ and $l$ is

$$
\frac{1}{2}\left[V \delta^{i, i+l} \mathbf{w}+W \delta^{i, i+l} \mathbf{v}\right]=\left[0, \cdots, 0, \tilde{f}^{i, i+l}, 0, \cdots, 0,-\tilde{f}^{i, i+l}, 0, \ldots, 0\right]^{T},
$$

where

$$
\tilde{f}^{i, i+l}=u_{i} v_{i+l}+v_{i} u_{i+l}
$$

is the product rule flux. Note that $(l-1)$ zeros separate the positive and negative flux values. Define the vector $\mathbf{1}^{i, l}$ as

$$
\mathbf{1}^{i, l}=\left[0, \cdots, 0,\left[\tilde{\mathbf{1}}^{i, l}\right]^{T}, 0, \cdots, 0\right]^{T}
$$

where $\left[\tilde{\mathbf{1}}^{i, l}\right]^{T}=[1, \cdots, 1]$, (a vector of ones of length $l$ ) begins at the $(i+1)$ location in $\mathbf{1}^{i, l}$. A very simple manipulation reveals,

$$
\frac{1}{2}\left[V \delta^{i, i+l} \mathbf{w}+W \delta^{i, i+l} \mathbf{v}\right]=\Delta \tilde{f}^{i, i+l} \mathbf{1}^{i, l}
$$


Accounting for all values of the parameters $i$ and $l$, and multiplying through by $\mathcal{P}^{-1}$ yields the desired result

$$
\left(V \mathcal{P}^{-1} \mathcal{Q} \mathbf{w}+W \mathcal{P}^{-1} \mathcal{Q} \mathbf{v}\right)=\mathcal{P}^{-1} \Delta\left[\tilde{\mathcal{B}} V \mathbf{w}+\sum_{i=1}^{N-1} \sum_{l=1}^{r(i)} q_{i, i+l} \tilde{f}^{i, i+l} \mathbf{1}^{i, l}\right]=\mathcal{P}^{-1} \Delta \overline{\mathbf{f}}_{e}
$$

Remark: The $\mathbf{1}^{i, l}$ vector in $\sum_{l=1}^{r(i)} q_{i, i+l} \tilde{f}^{i, i+l} \mathbf{1}^{i, l}$ spreads each $\tilde{f}^{i, i+l}$ to its neighbors on the interval $[i, i+l]$. Conversely, each flux $\overline{\mathbf{f}}_{i}$ receives contributions to from all its neighbors in the interval $[i-l, i]$.

Theorem 3.2. The local fluxes $\overline{\mathbf{f}}_{i}$ that result from the discrete split-form operator

$$
\alpha \mathcal{P}^{-1} \mathcal{Q} W \mathbf{v}+(1-\alpha)\left(V \mathcal{P}^{-1} \mathcal{Q} \mathbf{w}+W \mathcal{P}^{-1} \mathcal{Q} \mathbf{v}\right)=\mathcal{P}^{-1} \Delta \overline{\mathbf{f}}
$$

have compact support and are consistent with the original conservative flux $f(q)$. Thus, the Lax-Wendroff theorem guarantees that discretely captured discontinuities are accurate.

Proof. The compactness of $\overline{\mathbf{f}}_{e}$ follows immediately by inspection of eq. (3.8), as does the consistency of the flux for the divergence form $(\alpha=1)$. Considering the product rule term $(\alpha=0)$, substitute $w_{i}=w(q)$ and $v_{i}=v(q)$ into eq. (3.8) and sum over all indices to obtain the expression

$$
\begin{gathered}
\bar{f}_{i}=2 w v \sum_{k=1}^{r(i)} \sum_{l=1}^{k} q_{(i+l-k, i+l)} \\
1 \leq i+l, i+l-k \leq N \quad 1 \leq i \leq N-1, \\
\bar{f}_{0}=w v, \quad \bar{f}_{N}=w v
\end{gathered}
$$

where the additional $\sum_{l=1}^{k}$ in eq. (3.9) accounts for the contributions to each flux from $\sum_{l=1}^{r(i)} q_{i, i+l} \tilde{f}^{i, i+l} \mathbf{1}^{i, l}$. It is shown in lemma (2.5) that the double sum in eq. (3.9) satisfies the relation $\frac{1}{2}=\sum_{k=1}^{r(i)} \sum_{l=1}^{k} q_{(i+l-k, i+l)}$, which leads immediately to the desired result $\bar{f}_{i}=w v, \quad \forall \quad i$.

The two previous theorems are summarized as follows. The term $\alpha \mathcal{P}^{-1} \mathcal{Q} W \mathbf{v}+(1-\alpha)\left(V \mathcal{P}^{-1} \mathcal{Q} \mathbf{w}+W \mathcal{P}^{-1} \mathcal{Q} \mathbf{v}\right)$ can be expressed in terms of a telescoping conservative flux (in the $\mathcal{P}$-norm), has a compact support of halfwidth $r$, and is consistent with the original conservative flux in the conservation law given by eq. (2.1). All of the sufficient conditions of the Lax-Wendroff theorem are met, so converged solutions using the above split operators are weak solutions to the conservation law.

\section{A Multidomain Approach for Alternative Operators}

While great flexibility is afforded by the potential use of alternative forms of the equations (e.g., skewsymmetric or other splittings), a systematic approach is needed to extend alternative uniform-grid single- 
domain, tensor-product operators to complex geometries. A simple approach is to adopt a general multidomain finite-difference discretization that incorporates a generalized curvilinear mesh in each domain.

\subsection{Multidomain Operators}

A major obstacle in the application of high-order methods to realistic problems is the development of a suitable grid around complex geometric features or in regions of strong gradients. (Constraining the grid to design-order smoothness severely complicates grid generation.) Multidomain techniques greatly simplify the grid generation process for complex configurations by breaking the geometry into the union of piecewise smooth quadrilateral (hexahedral) domains in two (three) dimensions.

Conventional high-order SBP finite-difference techniques naturally extend to multidomain discretizations $[23,26,27,28]$. Each domain is discretized with a stable tensor-product formulation and then connected to its adjoining neighbors using interface conditions that maintain the stability, accuracy, and conservation of the interior operators. Penalty type interface treatments, which are closely related to those that are used in discontinuous Galerkin and internal penalty finite-element approaches, are most frequently used to join the domains. Domain interfaces need only be $C_{0}$ smooth to maintain the stability, conservation and design accuracy of the single-domain operators.

Existing derivations for multidomain high-order finite-difference schemes primarily focus on the divergence form of the equations. Indeed, stability and conservation proofs follow naturally with the use of this form. However, nothing precludes the use of alternative operators in each subdomain. The only additional requirement is the need for domain-interface coupling conditions that retain the desirable interior properties of the alternative operator. These interface operators must be developed on a case-by-case basis; however, as the examples in the next section demonstrate, these operators generally are simple extensions of the original coupling conditions introduced and extended in refs. [23, 26].

\subsection{Curvilinear Coordinates}

Generalized curvilinear coordinate formulations are commonly used in high-order formulations to facilitate the use of nonuniform grid distributions or the definition of "complex" geometries. The likelihood exists that the desirable attributes of a Cartesian grid, alternative formulation will not survive the curvilinear mappings between the physical $(x, y, z)$ and the computational $(\xi, \eta, \zeta)$ space. However, the curvilinear alternative formulation can inherit the desirable Cartesian grid properties. For example, Pirozzoli [16] recently demonstrated that pseudo-kinetic energy can be preserved on curvilinear grids that use an alternative (skew-symmetric) formulation, (i.e., one motivated by formulations that preserve kinetic energy on a Cartesian grid). 


\section{Examples using Burgers equation}

The conservation law form of the viscous Burgers equation is

$$
\begin{gathered}
u_{t}+f(u)_{x}=\epsilon u_{x x}, \quad f(u)=\left(\frac{u^{2}}{2}\right), \quad x \in[0,1], \quad t \in[0, \infty) \\
\tilde{a}_{0} u(0, t)-\epsilon u_{x}(0, t)-g_{0}(t)=0, \quad \tilde{a}_{1} u(1, t)-\epsilon u_{x}(1, t)-g_{1}(t)=0 \\
\tilde{a}_{0}=\frac{u(0, t)+|u(0, t)|}{3}, \quad \tilde{a}_{1}=\frac{u(1, t)-|u(1, t)|}{3}
\end{gathered}
$$

where $\epsilon$ represents the diffusion coefficient. Unlike the Navier-Stokes (or Euler) equations, the viscous (inviscid) Burgers equation a can be manipulated into canonical form by $\alpha$-splitting the nonlinear flux $f(u)$. In the canonical form, continuous and semi-discrete energy estimates can be derived and rigorous boundary and interface conditions can be formulated. Furthermore, by virtue of theorems (3.1) and (3.2) captured shocks are guaranteed to be accurate. Thus, Burgers equation provides an ideal setting to test the numerical properties (accuracy and stability) of the alternative split operators that are developed in the previous sections. ${ }^{6}$

Before testing can commence, stable and accurate boundary / interface conditions must be developed for the finite-domain split-form operators constructed in the previous section. Expressing the Burgers equation in canonical form, allows boundary conditions to be developed (i.e. those presented in (5.1)) that ensure that discrete energy growth is only function of the boundary data. Furthermore, these boundary conditions satisfy the constraint that as $\epsilon \rightarrow 0$, they smoothly evolve into a form appropriate for the inviscid Burgers equation. (This accounts for the fundamentally different nature of the problem when $\epsilon=0$.)

The discrete boundary / interface condition are developed using standard energy techniques. First, an energy analysis of the continuous problem is performed. Then, discrete boundary conditions are developed that allow the semi-discrete energy to mimic the continuous energy. Finally, discrete interface conditions are developed.

A comparison is made between the fully conservation form and the split-form developed herein. While in principle any value of $\alpha$ is admissible, only one $\alpha$ yields the energy estimate. This value of $\alpha$ is used in the next section to evaluate advantages, if any, that are gained from using the discrete formulation that satisfies the energy estimate.

The remainder of this section is devoted to developing numerical boundary and interface conditions for the viscous Burgers equation.

\subsection{Energy Analysis of the Continuous Problem}

The properties and behavior of the inviscid portion of eq. (5.1) are covered in an energy analysis that is presented by Jameson[20]; we reproduce and supplement the essential results here for completeness. The

\footnotetext{
${ }^{6}$ Indeed, any equation that can be symmetrized into canonical form using an $\alpha$-splitting technique exhibits the aforementioned fortuitous properties, and therefore is a good candidate for testing.
} 
energy that is given in Burgers equation can be analyzed directly without a linearization. If we assume a smooth solution, then we can split the conservation form (eq. (5.1)) by the parameter $\alpha$ to obtain

$$
u_{t}+\alpha\left(\frac{u^{2}}{2}\right)_{x}+(1-\alpha) u u_{x}=\epsilon u_{x x}, \quad x \in[0, L], t \in[0, \infty)
$$

This equation is multiplied by $u$ and integrated over the interval $[0, L]$ to obtain the energy

$$
\begin{aligned}
\frac{1}{2} \frac{d}{d t}\|u\|^{2} & =-\int_{0}^{L}\left[\alpha u\left(\frac{u^{2}}{2}\right)_{x}+(1-\alpha) u^{2} u_{x}\right] \mathrm{d} x+\epsilon \int_{0}^{L} u u_{x x} \mathrm{~d} x \\
& =-\int_{0}^{L}\left[\frac{\alpha}{2}\left(u^{3}\right)_{x}+\left(1-\frac{3}{2} \alpha\right) u^{2} u_{x}\right] \mathrm{d} x+\epsilon \int_{0}^{L}\left[\left(u u_{x}\right)_{x}-u_{x} u_{x}\right] \mathrm{d} x \\
& =\left.\frac{\alpha}{2} u^{3}\right|_{L} ^{0}+\left.\epsilon u u_{x}\right|_{0} ^{L}-\int_{0}^{L}\left(1-\frac{3}{2} \alpha\right) u^{2} u_{x} \mathrm{~d} x-\epsilon\left\|u_{x}\right\|^{2}
\end{aligned}
$$

Equation 5.3 clearly shows that for $\alpha=2 / 3$ the integral term disappears and the energy is dependent on the boundary data and the viscous dissipation. This is referred to as the canonical splitting or "skew-symmetric form" of the Burgers equation [20, 29]. For the viscous problem $(\epsilon>0)$, note that any shock that develops is resolvable at the continuous level and that the energy analysis remains valid. The energy stability of the boundary data is evaluated first by substituting $\alpha=2 / 3$ into eq. (5.3) and then by analyzing each boundary individually. The left boundary term (scaled by 2 for convenience) is

$$
B T_{0}=\frac{2}{3} u(0, t)^{3}-2 \epsilon u(0, t) u_{x}(0, t)
$$

Completing the squares in eq. 5.4 leads to boundary terms that can be written as

$$
B T_{0}=\frac{1}{\frac{2}{3} u(0, t)}\left\{\left[\frac{2}{3} u(0, t)^{2}-\epsilon u_{x}(0, t)\right]^{2}-\left(\epsilon u_{x}(0, t)\right)^{2}\right\}
$$

We define

$$
a(u)=\frac{2}{3} u(x, t)
$$

as a sensor for the hyperbolic part of the equation; $\tilde{a}_{0}$ and $\tilde{a}_{1}$ in the boundary conditions of eq. (5.1) serve as switches to eliminate the hyperbolic part of the boundary condition if waves are not propagating toward the interior of the domain from the boundaries. If we use the definition above, the left boundary term is

$$
B T_{0}=\frac{1}{a_{0}}\left\{\left[a_{0} u(0, t)-\epsilon u_{x}(0, t)\right]^{2}-\left[\epsilon u_{x}(0, t)\right]^{2}\right\}, \quad a_{0}=a[u(0, t)]
$$


We now substitute the left boundary condition from eq. (5.1) for $\epsilon u_{x}(0, t)$ in eq. (5.7) to obtain

$$
B T_{0}=\frac{1}{a_{0}}\left\{\left[a_{0} u(0, t)+g_{0}(t)-\tilde{a_{0}} u(0, t)\right]^{2}-\left[\tilde{a}_{0} u(0, t)-g_{0}(t)\right]^{2}\right\} .
$$

The condition $a_{0}>0$ in eq. (5.1) produces $\tilde{a}_{0}=a$, and we find that

$$
B T_{0}=\frac{g_{0}(t)^{2}}{a_{0}}-\frac{1}{a}\left[a_{0} u(0, t)-g_{0}(t)\right]^{2} \leq \frac{g_{0}(t)^{2}}{a_{0}}
$$

If $u(0, t)=a[u(0, t)]=0$, then $B T_{0}=0$, and the energy of the continuous equation is bounded from above by the boundary data. If $a<0$, then $\tilde{a}_{0}=0$, which yields the condition

$$
B T_{0}=\frac{1}{a_{0}}\left[a_{0} u(0, t)+g_{0}(t)\right]^{2}-\frac{g_{0}(t)^{2}}{a_{0}}=\frac{g_{0}(t)^{2}}{\left|a_{0}\right|}-\frac{1}{\left|a_{0}\right|}\left[a_{0} u(0, t)+g_{0}(t)\right]^{2}
$$

Again, the energy is bounded from above by the boundary data. The right boundary term (again scaled by $2)$ is

$$
B T_{1}=-\frac{2}{3} u(L, t)^{3}+2 \epsilon u(L, t) u_{x}(1, t)=-a_{1} u(L, t)^{2}+2 \tilde{a}_{1}-2 u(L, t) g_{1}(t)
$$

where $a_{1}=a[u(L, t)]$. The same procedure is followed to show that the energy on the right boundary is bounded. We find that

$$
B T_{1}= \begin{cases}0 & \text { for } \quad a_{1}=0 \\ \frac{g_{1}(t)^{2}}{a_{1}}-\frac{1}{a_{1}}\left[a_{1} u(L, t)+g_{1}(t)\right]^{2} & \text { for } \quad a_{1}>0 \\ \frac{g_{1}(t)^{2}}{\left|a_{1}\right|}-\frac{1}{\left|a_{1}\right|}\left[a_{1} u(L, t)-g_{1}(t)\right]^{2} & \text { for } \quad a_{1}<0\end{cases}
$$

The energy of the continuous equation is bounded by the energy imposed through the boundary terms.

\subsection{Energy Analysis of the Semidiscrete Problem: Single Domain}

The canonical splitting can be used to construct a semidiscrete operator that satisfies the semidiscrete analog to eq. (5.3). Using the finite-difference operators $\mathcal{D}$ and $\mathcal{D}_{2}$ that satisfy the $\mathrm{SBP}$ condition on the finite domain, we discretize the skew-symmetric form $(\alpha=2 / 3)$ of eq. (5.2) as

$$
\begin{gathered}
\mathbf{u}_{t}=-\frac{1}{3}[\mathcal{D} U \mathbf{u}+U \mathcal{D} \mathbf{u}]+\epsilon \mathcal{D}_{2} \mathbf{u} \\
+\sigma_{0} \mathcal{P}^{-1} \mathbf{e}_{0}\left[\tilde{a}_{0} u_{1}-\epsilon(\mathcal{S} \mathbf{u})_{1}-g_{0}(t)\right] \\
+\sigma_{1} \mathcal{P}^{-1} \mathbf{e}_{1}\left[\tilde{a}_{1} u_{N}-\epsilon(\mathcal{S} \mathbf{u})_{N}-g_{1}(t)\right] \\
U=\operatorname{diag}(\mathbf{u}), \quad \mathbf{e}_{0}=(1,0, \ldots)^{T}, \quad \mathbf{e}_{1}=(\ldots, 0,1)^{T} \\
\tilde{a}_{0}=\frac{u_{1}+\left|u_{1}\right|}{3} \quad \tilde{a}_{1}=\frac{u_{N}-\left|u_{N}\right|}{3}
\end{gathered}
$$


The proper values of $\sigma_{0}$ and $\sigma_{1}$ are determined in the following energy analysis. Both forms of $\mathcal{D}_{2}$ in eq. (D.1) (see appendix Appendix D) satisfy the energy analysis, but we use the narrow stencil here for illustrative purposes. The simultaneous approximation term (SAT) penalty method [30] is used in eq. (5.13) to satisfy the mixed boundary conditions with specified boundary data. For the finite domain, a diagonal norm $\mathcal{P}$ is chosen to ensure that matrix multiplication of the norm and the nonlinear coefficient matrix commutes. This choice reduces the overall accuracy for hyperbolic problems to $(p+1)$, where $2 p$ is the internal accuracy and $p$ is the accuracy at the boundary. For parabolic problems, the narrow-stencil second derivative operator $\mathcal{D}_{2}=\mathcal{P}^{-1}(\mathcal{R}+\mathcal{B S})$ constructed with the diagonal norm, provides a global $(p+2)$ order of accuracy [31]. Premultiplying eq. (5.13) by $\mathbf{u}^{T} \mathcal{P}$ and using eq. (2.2) yields the discrete energy

$$
\begin{aligned}
\frac{1}{2} \frac{d}{d t}\|\mathbf{u}\|_{\mathcal{P}}^{2}= & -\frac{1}{3} \mathbf{u}^{T}(\mathcal{Q} U+U \mathcal{Q}) \mathbf{u}+\epsilon \mathbf{u}^{T} \mathcal{R} \mathbf{u}+\epsilon \mathbf{u}^{T} \mathcal{B S} \mathbf{u} \\
& +\mathbf{u}^{T} \mathbf{e}_{0}\left\{\sigma_{0}\left[\tilde{a}_{0} u_{1}-\epsilon(\mathcal{S} \mathbf{u})_{1}-g_{0}(t)\right]\right\} \\
& +\mathbf{u}^{T} \mathbf{e}_{1}\left\{\sigma_{1}\left[\tilde{a}_{1} u_{N}-\epsilon(\mathcal{S} \mathbf{u})_{N}-g_{1}(t)\right]\right\}
\end{aligned}
$$

Adding eq. (5.14) to its transpose yields

$$
\begin{aligned}
\frac{d}{d t}\|\mathbf{u}\|_{\mathcal{P}}^{2}= & -\frac{2}{3} \mathbf{u}^{T} \mathcal{B} U \mathbf{u}+2 \epsilon \mathbf{u}^{T} \mathcal{R} \mathbf{u}+2 \epsilon \mathbf{u}^{T} \mathcal{B S} \mathbf{u} \\
& +2 u_{1} \sigma_{0}\left(\tilde{a}_{0} u_{1}-\epsilon[\mathcal{S} \mathbf{u})_{1}-g_{0}(t)\right] \\
& +2 u_{N} \sigma_{1}\left(\tilde{a}_{1} u_{N}-\epsilon[\mathcal{S} \mathbf{u})_{N}-g_{1}(t)\right]
\end{aligned}
$$

Simplifying with eq. (2.2) yields

$$
\begin{aligned}
\frac{d}{d t}\|\mathbf{u}\|_{\mathcal{P}}^{2}= & 2 \epsilon \mathbf{u}^{T} \mathcal{R} \mathbf{u} \\
& +2 u_{1}\left\{\sigma_{0}\left[\tilde{a}_{0} u_{1}-g_{0}(t)\right]+\frac{1}{3} u_{1}^{2}\right\}-2 \epsilon u_{1}\left[\left(\sigma_{0}+1\right)(\mathcal{S} \mathbf{u})_{1}\right] \\
& +2 u_{N}\left\{\sigma_{1}\left[\tilde{a}_{1} u_{N}-g_{1}(t)\right]-\frac{1}{3} u_{N}^{2}\right\}+2 \epsilon u_{N}\left[\left(-\sigma_{1}+1\right)(\mathcal{S} \mathbf{u})_{N}\right]
\end{aligned}
$$

Above, $\mathcal{R}$ is negative semidefinite and ensures that the energy only decays. The viscous boundary terms cancel for $\sigma_{0}=-1$ and $\sigma_{1}=1$, which leaves

$$
\begin{aligned}
\frac{d}{d t}\|\mathbf{u}\|_{\mathcal{P}}^{2} & =2 \epsilon \mathbf{u}^{T} \mathcal{R} \mathbf{u}+\widetilde{B T}_{0}+\widetilde{B T}_{1} \\
\widetilde{B T}_{0} & =u_{1}\left[a\left(u_{1}\right) u_{1}-2 \tilde{a}_{0} u_{1}+2 g_{0}(t)\right] \\
\widetilde{B T}_{1} & =u_{N}\left[2 \tilde{a}_{1} u_{N}-a\left(u_{N}\right) u_{N}-2 g_{1}(t)\right]
\end{aligned}
$$


For the discrete equation to mimic the continuous equation, the discrete energy must be bounded from above by the imposed boundary data. For the left boundary when $a\left(u_{1}\right) \geq 0$ and using completing the squares,

$$
\widetilde{B T}_{0}=-a u_{1}^{2}+2 u_{1} g_{0}=\frac{g_{0}^{2}}{a}-\frac{1}{a}\left(a u_{1}-g_{0}\right)^{2}
$$

The result in eq. 5.18 is equivalent to continuous result found in eq. (5.9).

Completing the squares at the left boundary if $a\left(u_{1}\right)<0$ yields

$$
\widetilde{B T}_{0}=a u_{1}^{2}+2 u_{1} g_{0}=\frac{g_{0}^{2}}{|a|}-\frac{1}{|a|}\left(a u_{1}+g_{0}\right)^{2}
$$

Again, note the similarity between eq. 5.19 and the continuous result given in eq. (5.10). The right boundary also mimics the continuous case. Note that the same penalty is used for the inviscid and the viscous Burgers equations.

\subsection{Energy Analysis of the Semidiscrete Problem: Multidomain}

Often multiple semidiscrete domains can be used advantageously to discretize a conservation law. Interface penalties are derived such that the semidiscrete energy is unaffected by the exchange of information between two domains. For a two domain problem on the interval $x \in[-1,1]$ divided at $x=b$, we use the discretization

$$
\begin{aligned}
\mathbf{x}^{(1)} & =\left[x_{1}^{(1)}, x_{2}^{(1)}, \ldots, x_{N_{1}-1}^{(1)}, x_{N_{1}}^{(1)}\right], \quad x_{i}^{(1)}=-1+(i-1) \frac{b+1}{N_{1}-1}, \quad i=1,2, \ldots, N_{1} \\
\mathbf{x}^{(2)} & =\left[x_{1}^{(2)}, x_{2}^{(2)}, \ldots, x_{N_{2}-1}^{(2)}, x_{N_{2}}^{(2)}\right], \quad x_{i}^{(2)}=b+(i-1) \frac{1-b}{N_{2}-1}, \quad i=1,2, \ldots, N_{2}
\end{aligned}
$$

where $N_{1}$ and $N_{2}$ are the number of uniform cells in each of the two domains. The solution on each domain is denoted by $\mathbf{u}^{(1)}$ and $\mathbf{u}^{(2)}$, respectively. Operators that are defined on each domain are denoted similarly. The semidiscrete Burgers equation for the multidomain problem is

$$
\begin{aligned}
& \mathbf{u}_{t}^{(1)}=-\frac{1}{3}\left(\mathcal{D}^{(1)} U^{(1)} \mathbf{u}^{(1)}+U^{(1)} \mathcal{D}^{(1)} \mathbf{u}^{(1)}\right)+\epsilon \mathcal{D}_{2}^{(1)} \mathbf{u}^{(1)} \\
&+\left(\mathcal{P}^{(1)}\right)^{-1} \mathbf{e}_{1}^{(1)}\left\{\left(k_{00} u_{N_{1}}^{(1)}-k_{01} u_{1}^{(2)}\right)+l_{00} \epsilon\left(u_{N_{1}}^{(1)}-u_{1}^{(2)}\right)\right. \\
&\left.+\epsilon l_{01}\left[\left(\mathcal{S}^{(1)} \mathbf{u}^{(1)}\right)_{N_{1}}-\left(\mathcal{S}^{(2)} \mathbf{u}^{(2)}\right)_{1}\right]\right\} \\
& \mathbf{u}_{t}^{(2)}=-\frac{1}{3}\left(\mathcal{D}^{(2)} U^{(2)} \mathbf{u}^{(2)}+U^{(2)} \mathcal{D}^{(2)} \mathbf{u}^{(2)}\right)+\epsilon \mathcal{D}_{2}^{(2)} \mathbf{u}^{(2)} \\
&+\left(\mathcal{P}^{(2)}\right)^{-1} \mathbf{e}_{0}^{(2)}\left\{\left(k_{11} u_{1}^{(2)}-k_{10} u_{N_{1}}^{(1)}\right)+r_{00} \epsilon\left(u_{1}^{(2)}-u_{N_{1}}^{(1)}\right)\right. \\
&\left.+\epsilon r_{01}\left[\left(\mathcal{S}^{(2)} \mathbf{u}^{(2)}\right)_{1}-\left(\mathcal{S}^{(1)} \mathbf{u}^{(1)}\right)_{N_{1}}\right]\right\} \\
& U^{(1)}=\operatorname{diag}\left(\mathbf{u}^{(1)}\right), \quad U^{(2)}=\operatorname{diag}\left(\mathbf{u}^{(2)}\right), \quad \mathbf{e}_{1}^{(1)}=(\ldots, 0,1)^{T}, \quad \mathbf{e}_{0}^{(2)}=(1,0, \ldots)^{T}
\end{aligned}
$$


where the boundary condition penalties have been dropped for clarity of the interface treatment. The energy is calculated by premultiplying the two equations by $\left(\mathbf{u}^{(1)}\right)^{T} \mathcal{P}^{(1)}$ and $\left(\mathbf{u}^{(2)}\right)^{T} \mathcal{P}^{(2)}$, respectively. The equations are then added to their transpose as

$$
\begin{aligned}
& \frac{d}{d t}\left(\left\|\mathbf{u}^{(1)}\right\|_{\mathcal{P}^{(1)}}^{2}+\left\|\mathbf{u}^{(2)}\right\|_{\mathcal{P}^{(2)}}^{2}\right)+2 \epsilon\left(\left\|\mathbf{u}_{x}^{(1)}\right\|_{\mathcal{P}^{(1)}}^{2}+\left\|\mathbf{u}_{x}^{(2)}\right\|_{\mathcal{P}^{(2)}}^{2}\right) \\
& =\frac{2}{3}\left[\left(u_{1}^{(2)}\right)^{3}-\left(u_{N_{1}}^{(1)}\right)^{3}\right]+2 \epsilon\left[\left(S^{(1)} \mathbf{u}^{(1)}\right)_{N_{1}}-\left(S^{(2)} \mathbf{u}^{(2)}\right)_{1}\right] \\
& +2 u_{N_{1}}^{(1)}\left\{\left(k_{00} u_{N_{1}}^{(1)}-k_{01} u_{1}^{(2)}\right)+l_{00} \epsilon\left(u_{N_{1}}^{(1)}-u_{1}^{(2)}\right)\right. \\
& \left.+\epsilon l_{01}\left[\left(\mathcal{S}^{(1)} \mathbf{u}^{(1)}\right)_{N_{1}}-\left(\mathcal{S}^{(2)} \mathbf{u}^{(2)}\right)_{1}\right]\right\} \\
& +2 u_{1}^{(2)}\left\{\left(k_{11} u_{1}^{(2)}-k_{10} u_{N_{1}}^{(1)}\right)+r_{00} \epsilon\left(u_{1}^{(2)}-u_{N_{1}}^{(1)}\right)\right. \\
& \left.+\epsilon r_{01}\left[\left(\mathcal{S}^{(2)} \mathbf{u}^{(2)}\right)_{1}-\left(\mathcal{S}^{(1)} \mathbf{u}^{(1)}\right)_{N_{1}}\right]\right\}
\end{aligned}
$$

The terms that are related to the boundary data have been removed. We seek inviscid terms that do not contribute to the energy and viscous terms that are guaranteed to be stable and also satisfy the weak form. The derivation of the viscous terms is given in Carpenter, Nordström, and Gottlieb [24]. The conservation requirement is

$$
\begin{gathered}
k_{00}=\frac{1}{3} u_{N_{1}}^{(1)}, \quad k_{10}=-k_{01}, \quad k_{11}=-\frac{1}{3} u_{1}^{(2)} \\
l_{00}=r_{00}, \quad r_{01}=l_{01}+1 .
\end{gathered}
$$

The formal order of accuracy of the penalty terms must be the same as the order of the boundary finitedifference approximations. This requires that the value of $k_{01}$ be a function of $\mathbf{u}$. We choose an equal weighting of the values on either side of the interface:

$$
k_{01}=\frac{u_{N_{1}}^{(1)}+u_{1}^{(2)}}{2} .
$$

The following coefficients satisfy the sufficient conditions for stability of the viscous penalty:

$$
l_{00}=-\frac{1}{4\left(\alpha^{(1)}+\alpha^{(2)}\right)}, \quad l_{01}=-\frac{\alpha^{(2)}}{\alpha^{(1)}+\alpha^{(2)}}, \quad \alpha^{(1)}=\mathcal{P}_{\left(N_{1}\right)\left(N_{1}\right)}^{(1)}, \quad \alpha^{(2)}=\mathcal{P}_{(1)(1)}^{(2)}
$$

where $\alpha^{(1)}$ and $\alpha^{(2)}$ are "borrowed" from $\epsilon\left(\left\|\mathbf{u}_{x}^{(1)}\right\|_{\mathcal{P}^{(1)}}^{2}+\left\|\mathbf{u}_{x}^{(2)}\right\|_{\mathcal{P}^{(2)}}^{2}\right)$ [24]. In this form, the interface will only dissipate energy for viscous problems. For inviscid problems, the energy is dependent only on the initial and boundary conditions.

\section{Numerical Tests}

The new discrete operators are implemented first for the Burgers equation in a one-dimensional finitedifference solver with a uniform grid. Integration in time is conducted with a five-step, fourth-order Runge- 
Kutta scheme [32]. The step size in time is chosen such that the temporal error is negligible in comparison with the spatial error. Three problems are evaluated. For the first problem, the accuracy of the three operator sets that are developed herein is tested by using the skew-symmetric $(\alpha=2 / 3)$ and the conservation $(\alpha=1)$ forms of the viscous Burgers equation. The second problem tests the accuracy of the multidomain interface closures that are developed based on the new operator sets. The final problem is derived from the Euler equations of gas dynamics, whereby it is established that split forms of the Euler equations predict the correct shock locations.

\subsection{Accuracy Validation}

The viscous Burgers equation is used to test the accuracy of the new operator when it is applied to the nonlinear equation. The test problem is defined as

$$
\begin{aligned}
\mathbf{u}_{t} & =\mathcal{P}^{-1} \Delta\left(\alpha \overline{\mathbf{f}}_{c}+(1-\alpha) \overline{\mathbf{f}}_{e}+\overline{\mathbf{f}}_{V}\right) \\
& +\sigma_{0} \mathcal{P}^{-1} \mathbf{e}_{0}\left[-\tilde{a}_{0} u_{1}-\epsilon(\mathcal{S} \mathbf{u})_{1}-g_{0}(t)\right] \\
& +\sigma_{1} \mathcal{P}^{-1} \mathbf{e}_{1}\left[-\tilde{a}_{1} u_{N}-\epsilon(\mathcal{S} \mathbf{u})_{N}-g_{1}(t)\right] \\
g_{0}(t) & =\frac{v(-1, t)+|v(-1, t)|}{3} v(-1, t)-\epsilon v_{x}(-1, t) \\
g_{1}(t) & =\frac{v(1, t)-|v(1, t)|}{3} v(1, t)-\epsilon v_{x}(1, t) \\
v(x, t) & =\frac{4 x}{x^{2}+2 t+\frac{1}{40}}, \quad x \in\left[x_{1}, x_{N}\right], \quad t \in[0, T]
\end{aligned}
$$

where $\epsilon=1$ and the initial condition is $\mathbf{u}_{0}=v(\mathbf{x}, 0)$. The definition of the viscous flux $\overline{\mathbf{f}}_{V}$ is given in Appendix D. The values for the penalty coefficients $\sigma$ are those that are identified in section 5 . Note that the sign of the convective term has changed for this problem. This is to satisfy the exact solution $v(x, t)$. The problem is simulated on the interval $[-1,1]$ up to $T=0.05$. Five grids are used to determine the order of accuracy of the set of operators. The $L^{2}$ and $L^{\infty}$ error norms of the conservation form and the canonical split-form are compared in tables 2 through 4 . Figure 1 shows the numerical solution for $N=64$ at different times. All simulations use the narrow-stencil viscous operator.

Table 2: Error Norms and Convergence Rates for Viscous Burgers Equation Using Canonical Split and Conservation Forms, and Conservative Narrow-stencil (2-4-2) Viscous Operator.

\begin{tabular}{c|cccc|cccc} 
& \multicolumn{3}{|c|}{ Canonical Split } & \multicolumn{4}{c}{ Conservation } \\
\hline$N$ & $L^{2}$ Error & $L^{2}$ Rate & $L^{\infty}$ Error & $L^{\infty}$ Rate & $L^{2}$ Error & $L^{2}$ Rate & $L^{\infty}$ Error & $L^{\infty}$ Rate \\
\hline 32 & $1.64 \mathrm{e}-03$ & - & $2.70 \mathrm{e}-03$ & - & $1.29 \mathrm{e}-03$ & - & $2.44 \mathrm{e}-03$ & - \\
64 & $1.10 \mathrm{e}-04$ & 3.90 & $1.91 \mathrm{e}-04$ & 3.82 & $8.86 \mathrm{e}-05$ & 3.86 & $1.75 \mathrm{e}-04$ & 3.80 \\
128 & $6.97 \mathrm{e}-06$ & 3.98 & $1.21 \mathrm{e}-05$ & 3.97 & $5.66 \mathrm{e}-06$ & 3.97 & $1.17 \mathrm{e}-05$ & 3.90 \\
256 & $4.38 \mathrm{e}-07$ & 3.99 & $7.66 \mathrm{e}-07$ & 3.99 & $3.56 \mathrm{e}-07$ & 3.99 & $7.38 \mathrm{e}-07$ & 3.99 \\
512 & $2.74 \mathrm{e}-08$ & 4.00 & $4.79 \mathrm{e}-08$ & 4.00 & $2.23 \mathrm{e}-08$ & 4.00 & $4.62 \mathrm{e}-08$ & 4.00
\end{tabular}

The expected order of accuracy is $p+2$, where $p$ is the boundary accuracy. This design order is achieved for both the skew-symmetric and conservation forms for the (2-4-2) operator. However, the (3-6-3) and (4-84) operators exhibit greater accuracy than design order convergence. The (2-4-2) operator does not deviate 
Table 3: Error Norms and Convergence Rates for Viscous Burgers Equation Using Canonical Split and Conservation Forms, and Conservative Narrow-stencil (3-6-3) Viscous Operator.

\begin{tabular}{c|cccc|cccc} 
& \multicolumn{4}{|c|}{ Canonical Split } & \multicolumn{5}{c}{ Conservation } \\
\hline$N$ & $L^{2}$ Error & $L^{2}$ Rate & $L^{\infty}$ Error & $L^{\infty}$ Rate & $L^{2}$ Error & $L^{2}$ Rate & $L^{\infty}$ Error & $L^{\infty}$ Rate \\
\hline 32 & $2.34 \mathrm{e}-04$ & - & $4.62 \mathrm{e}-04$ & - & $2.83 \mathrm{e}-04$ & - & $6.24 \mathrm{e}-04$ & - \\
64 & $4.56 \mathrm{e}-06$ & 5.69 & $9.15 \mathrm{e}-06$ & 5.66 & $5.61 \mathrm{e}-06$ & 5.65 & $1.30 \mathrm{e}-05$ & 5.58 \\
128 & $7.52 \mathrm{e}-08$ & 5.92 & $1.51 \mathrm{e}-07$ & 5.92 & $9.31 \mathrm{e}-08$ & 5.91 & $2.19 \mathrm{e}-07$ & 5.90 \\
256 & $1.19 \mathrm{e}-09$ & 5.98 & $2.40 \mathrm{e}-09$ & 5.98 & $1.48 \mathrm{e}-09$ & 5.98 & $3.49 \mathrm{e}-09$ & 5.97 \\
512 & $2.00 \mathrm{e}-11$ & 5.90 & $3.98 \mathrm{e}-11$ & 5.92 & $2.33 \mathrm{e}-11$ & 5.99 & $5.37 \mathrm{e}-11$ & 6.02
\end{tabular}

Table 4: Error Norms and Convergence Rates for Viscous Burgers Equation Using Canonical Split and Conservation Forms, and Conservative Narrow-stencil (4-8-4) Viscous Operator.

\begin{tabular}{c|cccc|cccc} 
& \multicolumn{5}{|c|}{ Canonical Split } & \multicolumn{5}{c}{ Conservation } \\
\hline$N$ & $L^{2}$ Error & $L^{2}$ Rate & $L^{\infty}$ Error & $L^{\infty}$ Rate & $L^{2}$ Error & $L^{2}$ Rate & $L^{\infty}$ Error & $L^{\infty}$ \\
\hline 32 & $1.52 \mathrm{e}-04$ & - & $3.64 \mathrm{e}-04$ & - & $1.89 \mathrm{e}-04$ & - & $4.42 \mathrm{e}-04$ & - \\
64 & $6.97 \mathrm{e}-07$ & 7.77 & $2.17 \mathrm{e}-06$ & 7.39 & $8.58 \mathrm{e}-07$ & 7.78 & $2.46 \mathrm{e}-06$ & 7.49 \\
128 & $3.85 \mathrm{e}-09$ & 7.50 & $1.78 \mathrm{e}-08$ & 6.93 & $4.45 \mathrm{e}-09$ & 7.59 & $1.92 \mathrm{e}-08$ & 7.00 \\
256 & $2.74 \mathrm{e}-11$ & 7.13 & $1.91 \mathrm{e}-10$ & 6.55 & $2.94 \mathrm{e}-11$ & 7.24 & $1.98 \mathrm{e}-10$ & 6.60 \\
512 & $1.74 \mathrm{e}-12$ & 3.98 & $2.65 \mathrm{e}-12$ & 6.17 & $1.73 \mathrm{e}-12$ & 4.09 & $2.69 \mathrm{e}-12$ & 6.20
\end{tabular}

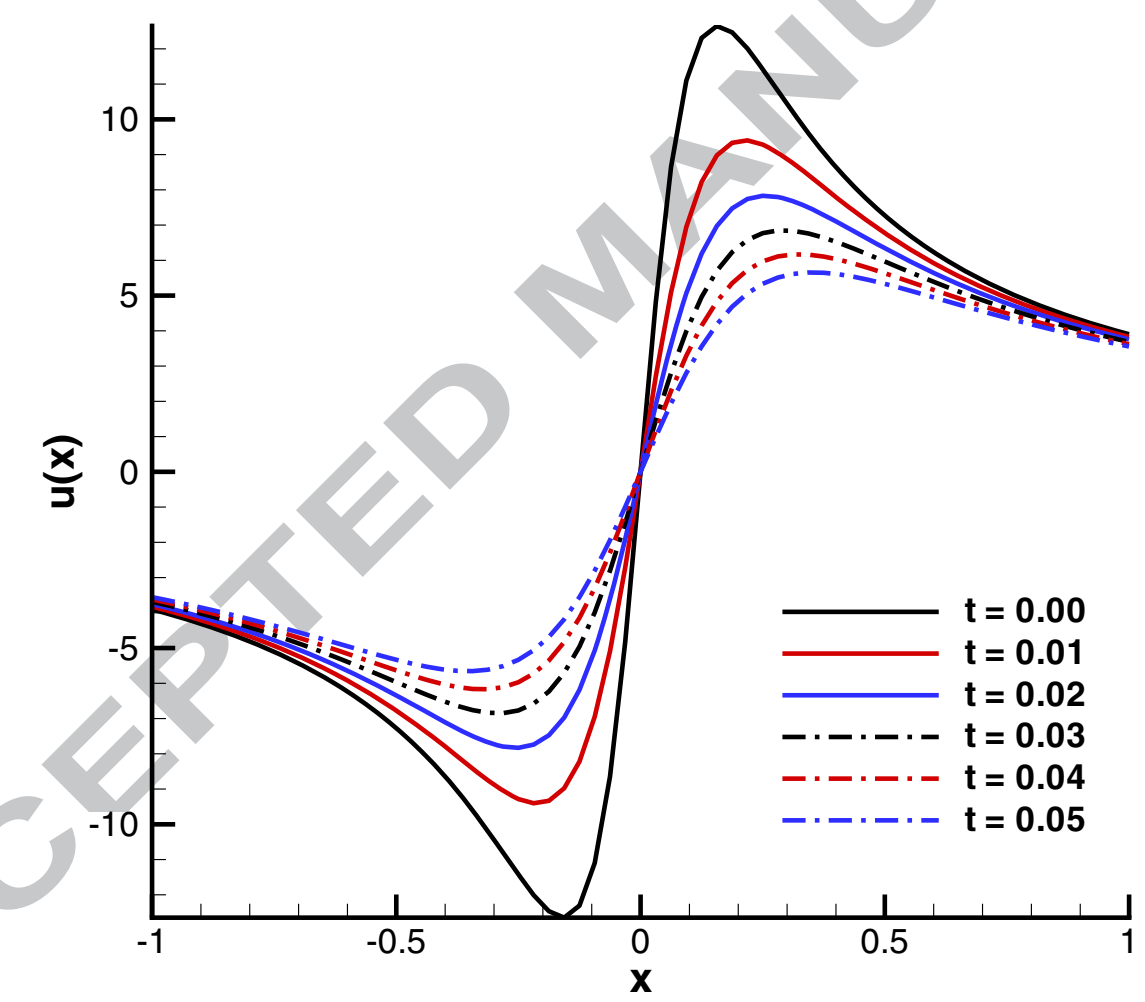

Figure 1: Numerical approximation to skew-symmetric form of viscous Burgers equation with 64 uniform cells and the (4-8-4) narrow stencil telescoping operators.

from design order because the maximum order that can be achieved is equivalent to the design order. This is not the case for the (3-6-3) and (4-8-4) operators. For these operators, the accuracy is at least design order for well resolved solutions. For the (2-4-2) operator, the conservation form is slightly more accurate than the canonical split-form. For the (3-6-3) and (4-8-4) operators, the canonical split-form is more accurate. 


\subsection{Multidomain Validation}

The test problem that is used to test the interfaces is described by the exact solution

$$
v(x, t)=1-\tanh \left(\frac{x-t-x_{0}}{2 \epsilon}\right), \quad x \in[-10,10], \quad t \in[0, T]
$$

where $\epsilon=0.25, x_{0}=-5.0$, and the initial condition is $\mathbf{u}_{0}=v(\mathbf{x}, 0)$. The problem is simulated up to $T=10.0$ with four grid resolutions. The skew-symmetric form of the discrete equations is used.

The $L^{2}$ and $L^{\infty}$ errors are tabulated in tables 5 through 7 for each operator on (1) a single domain, (2) two domains with equivalent spacing, and (3) two domains with grid spacing that changes discontinuously at the interface by a factor of $r=2$. (Herein, the jump in spacing is denoted as the "compression ratio.") The interface between domains is located at $x=0.0$. The discrete solution with 256 uniform cells on a single domain for the (2-4-2) operator set is shown in figure 2.

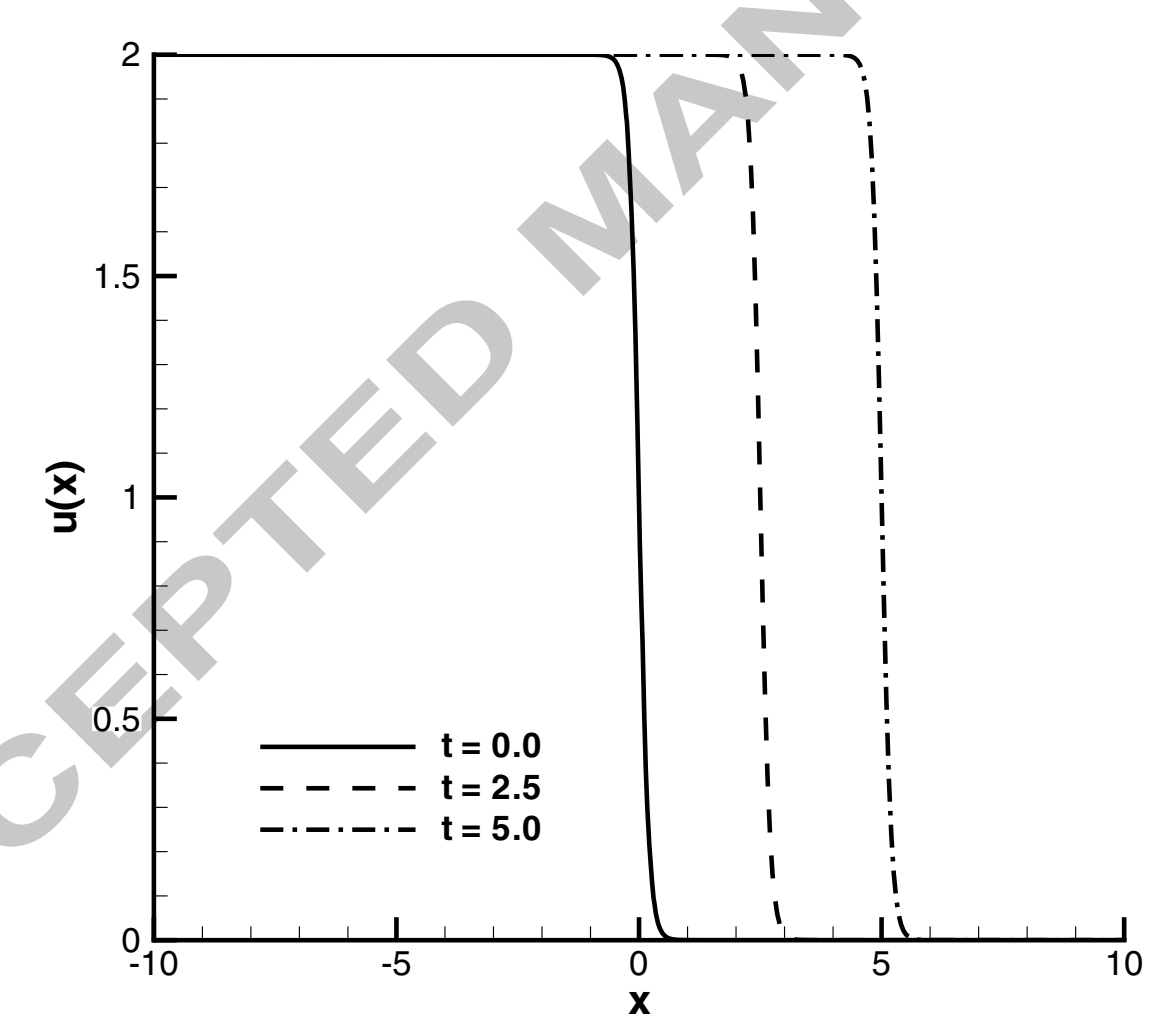

Figure 2: Discrete solution to viscous Burgers equation using 256 equispaced nodes with (4-8-4) narrow operators.

Tables 5 through 7 demonstrate that the interface has a negligible effect on the accuracy for this wellresolved test case. In addition, the treatment has been verified to be design order accurate, even when a discontinuous grid resolution is used across the subdomains. 
Table 5: Error Norms and Convergence Rates of (2-4-2) Operator for Single Domain, Two Domains with Uniform Resolution, and Two Domains with compression ratio $r=2$.

\begin{tabular}{|c|c|c|c|c|c|}
\hline \multicolumn{6}{|c|}{ Single Domain } \\
\hline \multirow{2}{*}{\multicolumn{2}{|c|}{$\begin{array}{c}\text { Resolution } \\
N\end{array}$}} & \multicolumn{4}{|c|}{ Error } \\
\hline & & $L^{2}$ Error & $L^{2}$ Rate & $L^{\infty}$ Error & $L^{\infty}$ Rate \\
\hline \multicolumn{2}{|c|}{64} & $8.89 \mathrm{e}-03$ & - & $1.13 \mathrm{e}-02$ & - \\
\hline \multicolumn{2}{|c|}{128} & $6.37 \mathrm{e}-04$ & 3.80 & $8.86 \mathrm{e}-04$ & 3.68 \\
\hline \multicolumn{2}{|c|}{256} & $4.19 \mathrm{e}-05$ & 3.93 & $6.17 \mathrm{e}-05$ & 3.84 \\
\hline \multicolumn{2}{|c|}{512} & $2.66 \mathrm{e}-06$ & 3.98 & $4.04 \mathrm{e}-06$ & 3.93 \\
\hline \multicolumn{6}{|c|}{ Two Domains, $r=1$} \\
\hline \multicolumn{2}{|c|}{ Resolution } & \multicolumn{4}{|c|}{ Error } \\
\hline$N_{1}$ & $N_{2}$ & $L^{2}$ Error & $L^{2}$ Rate & $L^{\infty}$ Error & $L^{\infty}$ Rate \\
\hline 32 & 32 & $8.91 \mathrm{e}-03$ & - & $1.14 \mathrm{e}-02$ & - \\
\hline 64 & 64 & $6.38 \mathrm{e}-04$ & 3.80 & $8.87 \mathrm{e}-04$ & 3.68 \\
\hline 128 & 128 & $4.19 \mathrm{e}-05$ & 3.93 & $6.17 \mathrm{e}-05$ & 3.85 \\
\hline 256 & 256 & $2.66 \mathrm{e}-06$ & 3.98 & $4.04 \mathrm{e}-06$ & 3.93 \\
\hline \multicolumn{6}{|c|}{ Two Domains, $r=2$} \\
\hline \multicolumn{2}{|c|}{ Resolution } & \multicolumn{4}{|c|}{ Error } \\
\hline$N_{1}$ & $N_{2}$ & $L^{2}$ Error & $L^{2}$ Rate & $L^{\infty}$ Error & $L^{\infty}$ Rate \\
\hline 32 & 64 & $6.26 \mathrm{e}-04$ & - & $8.65 \mathrm{e}-04$ & - \\
\hline 64 & 128 & $4.12 \mathrm{e}-05$ & 3.93 & $6.08 \mathrm{e}-05$ & \\
\hline 128 & 256 & $2.61 \mathrm{e}-06$ & 3.98 & $3.97 \mathrm{e}-06$ & 3.94 \\
\hline 256 & 512 & $1.64 \mathrm{e}-07$ & 4.00 & $2.50 \mathrm{e}-07$ & \\
\hline
\end{tabular}

Table 6: Error Norms and Convergence Rates of (3-6-3) Operator for Single Domain, Two Domains with Uniform Resolution, and Two Domains with compression ratio $r=2$.

\begin{tabular}{|c|c|c|c|c|c|}
\hline \multirow{2}{*}{\multicolumn{2}{|c|}{$\frac{\text { Resolution }}{N}$}} & \multicolumn{4}{|c|}{ Error } \\
\hline & & $L^{2}$ Error & $L^{2}$ Rate & $L^{\infty}$ Error & $L^{\infty}$ Rate \\
\hline \multicolumn{2}{|c|}{64} & $3.48 \mathrm{e}-03$ & $-3.96 \mathrm{e}-03$ & - & \\
\hline \multicolumn{2}{|c|}{128} & $9.88 \mathrm{e}-05$ & 5.14 & $1.33 \mathrm{e}-04$ & 4.89 \\
\hline \multicolumn{2}{|c|}{256} & $1.89 \mathrm{e}-06$ & 5.71 & $3.23 \mathrm{e}-06$ & 5.37 \\
\hline \multirow{2}{*}{\multicolumn{2}{|c|}{512}} & $3.13 \mathrm{e}-08$ & 5.92 & $5.41 \mathrm{e}-08$ & 5.90 \\
\hline & & \multicolumn{3}{|c|}{ Two Domains, $r=1$} & \\
\hline \multicolumn{2}{|c|}{ Resolution } & \multicolumn{3}{|c|}{ Error } & \\
\hline$N_{1}$ & $N_{2}$ & $L^{2}$ Error & $L^{2}$ Rate & $L^{\infty}$ Error & $L^{\infty}$ Rate \\
\hline 32 & 32 & $3.47 \mathrm{e}-03$ & - & $3.91 \mathrm{e}-03$ & - \\
\hline 64 & 64 & $9.87 \mathrm{e}-05$ & 5.14 & $1.33 \mathrm{e}-04$ & 4.88 \\
\hline 128 & 128 & $1.89 \mathrm{e}-06$ & 5.71 & $3.21 \mathrm{e}-06$ & 5.37 \\
\hline \multirow[t]{2}{*}{256} & 256 & $3.11 \mathrm{e}-08$ & 5.92 & $5.37 \mathrm{e}-08$ & 5.90 \\
\hline & & \multicolumn{3}{|c|}{ Two Domains, $r=2$} & \\
\hline \multicolumn{2}{|c|}{ Resolution } & \multicolumn{4}{|c|}{ Error } \\
\hline$N_{1}$ & $N_{2}$ & $L^{2}$ Error & $L^{2}$ Rate & $L^{\infty}$ Error & $L^{\infty}$ Rate \\
\hline 32 & 64 & $9.82 \mathrm{e}-05$ & - & $1.34 \mathrm{e}-04$ & - \\
\hline 64 & 128 & $1.87 \mathrm{e}-06$ & 5.72 & $3.15 \mathrm{e}-06$ & 5.41 \\
\hline 128 & 256 & $3.11 \mathrm{e}-08$ & 5.91 & $5.05 \mathrm{e}-08$ & 5.96 \\
\hline 256 & 512 & $5.54 \mathrm{e}-10$ & 5.81 & $7.55 \mathrm{e}-10$ & 6.07 \\
\hline
\end{tabular}

\subsection{Lax's Shock Tube Problem}

To demonstrate that the shock location is independent of the splitting parameter, a test was also conducted on Lax's shock tube problem for the compressible Euler equations. The details are provided below. The nondissipative divergence form is compared to the splitting of Honein and Moin [12], and it is demonstrated that the shock location is not altered by the splitting. To capture the shock, artificial dissipation was added in the form of artificial viscosity, applied in a manner consistent with the Lax Wendroff theorem.

The differential form of the one-dimensional Euler equations is

$$
q_{t}+f_{x}=0,
$$

where the conserved variables, $q=(\rho, \rho v, \rho E)^{T}$ are mass, momentum, and energy. The conservative flux is $f=(\rho v, \rho v v+p, \rho v E+p v)^{T}$. The viscous regularization that will be employed for these equations has the 
Table 7: Error Norms and Convergence Rates of (4-8-4) Operator for Single Domain, Two Domains with Uniform Resolution, and Two Domains with compression ratio $r=2$.

\begin{tabular}{|c|c|c|c|c|c|}
\hline \multicolumn{6}{|c|}{ Single Domain } \\
\hline \multirow{2}{*}{\multicolumn{2}{|c|}{$\frac{\text { Resolution }}{N}$}} & \multicolumn{4}{|c|}{ Error } \\
\hline & & $L^{2}$ Error & $L^{2}$ Rate & $L^{\infty}$ Error & $L^{\infty}$ Rate \\
\hline \multicolumn{2}{|c|}{64} & $1.97 \mathrm{e}-03$ & - & $2.00 \mathrm{e}-03$ & - \\
\hline \multicolumn{2}{|c|}{128} & $2.88 \mathrm{e}-05$ & 6.10 & $3.56 \mathrm{e}-05$ & 5.81 \\
\hline \multicolumn{2}{|c|}{256} & $1.81 \mathrm{e}-07$ & 7.31 & $3.37 \mathrm{e}-07$ & 6.72 \\
\hline \multicolumn{2}{|c|}{512} & $8.53 \mathrm{e}-10$ & 7.73 & $1.36 \mathrm{e}-09$ & 7.95 \\
\hline \multicolumn{6}{|c|}{ Two Domains, $r=1$} \\
\hline \multicolumn{2}{|c|}{ Resolution } & \multicolumn{4}{|c|}{ Error } \\
\hline$N_{1}$ & $N_{2}$ & $L^{2}$ Error & $L^{2}$ Rate & $L^{\infty}$ Error & $L^{\infty}$ Rate \\
\hline 32 & 32 & $2.16 \mathrm{e}-03$ & - & $2.70 \mathrm{e}-03$ & - \\
\hline 64 & 64 & $2.89 \mathrm{e}-05$ & 6.23 & $3.79 \mathrm{e}-05$ & 6.15 \\
\hline 128 & 128 & $1.84 \mathrm{e}-07$ & 7.29 & $2.93 \mathrm{e}-07$ & 7.02 \\
\hline 256 & 256 & $1.01 \mathrm{e}-09$ & 7.51 & $1.67 \mathrm{e}-09$ & 7.46 \\
\hline \multicolumn{6}{|c|}{ Two Domains, $r=2$} \\
\hline \multicolumn{2}{|c|}{$\overline{\text { Resolution }}$} & \multicolumn{4}{|c|}{ Error } \\
\hline$N_{1}$ & $N_{2}$ & $L^{2}$ Error & $L^{2}$ Rate & $L^{\infty}$ Error & $L^{\infty}$ Rate \\
\hline 32 & 64 & $3.01 \mathrm{e}-05$ & - & $4.44 \mathrm{e}-05$ & - \\
\hline 64 & 128 & $2.67 \mathrm{e}-07$ & 6.82 & $4.36 \mathrm{e}-07$ & 6.67 \\
\hline 128 & 256 & $3.43 \mathrm{e}-09$ & 6.28 & $4.63 \mathrm{e}-09$ & 6.56 \\
\hline 256 & 512 & $1.09 \mathrm{e}-10$ & 4.98 & $4.83 \mathrm{e}-11$ & \\
\hline
\end{tabular}

form

$$
f^{(v)}=\left(0, \mu u_{x}, \mu v v_{x}+\kappa T_{x}\right)^{T},
$$

where $\mu$ and $\kappa$ represent the dynamic viscosity and thermal conductivity, respectively.

The non-dissipative divergence form is calculated using,

$$
\mathcal{P}^{-1} \Delta \overline{\mathbf{f}}^{(d)}=\left(\begin{array}{c}
\mathcal{D}[\rho] \mathbf{v} \\
\mathcal{D}[\rho][v] \mathbf{v}+\mathcal{D} \mathbf{p} \\
\mathcal{D}[\rho][v] \mathbf{E}+\mathcal{D}[p] \mathbf{v}
\end{array}\right)
$$

where $[\rho],[v]$, and $[p]$ denote that the discrete values have been injected onto the diagonal of a matrix. This discretization form is well known to suffer from numerical instability for a wide range of problems without some form of added stabilization. Many alternative forms exist, but herein we show the form of Honein and Moin [12],

$$
\mathcal{P}^{-1} \Delta \overline{\mathbf{f}}^{(h m)}=\left(\begin{array}{c}
\mathcal{D}[\rho] \mathbf{v} \\
\frac{1}{2}(\mathcal{D}[\rho][v] \mathbf{v}+[\rho][v] \mathcal{D} \mathbf{v}+[v] \mathcal{D}[\rho] \mathbf{v})+\mathcal{D} \mathbf{p} \\
\frac{1}{2}(\mathcal{D}[\rho][v] \mathbf{e}+[\rho][v] \mathcal{D} \mathbf{e}+[e] \mathcal{D}[\rho] \mathbf{v})+\frac{1}{2}([v] \mathcal{D}[\rho][v] \mathbf{v}+[\rho][v][v] \mathcal{D} \mathbf{v})+[v] \mathcal{D} \mathbf{p}+[p] \mathcal{D} \mathbf{v}
\end{array}\right) .
$$

This form has been shown to exhibit better stability properties for underresolved flows. From the work herein, it should be immediately clear that this form can be transformed term-by-term into a telescopic flux form as long as $\mathcal{D}$ is a SBP operator with a diagonal norm.

An artificial viscosity method, based on the form of the regularization in eq. 6.4, is used to capture shocks. That is, the semi-discrete form being solved is

$$
\mathbf{u}_{t}+\mathcal{P}^{-1} \Delta\left(\overline{\mathbf{f}}-\overline{\mathbf{f}}^{(a v)}\right)=\mathbf{g}_{b}
$$


where $\mathbf{g}_{b}$ contains boundary penalties. The artificial viscosity takes the form,

$$
\mathcal{P}^{-1} \Delta \overline{\mathbf{f}}^{(a v)}=\left(\begin{array}{c}
\mathbf{0} \\
\mathcal{D}_{2}\left(\left[\mu_{c}\right]\right) \mathbf{v} \\
\mathcal{D}_{2}\left(\left[\mu_{c}\right][\mathbf{v}]\right) \mathbf{v}+\mathcal{D}_{2}\left(\left[\kappa_{c}\right]\right) \mathbf{T}
\end{array}\right)
$$

where $\mathcal{D}_{2}(\vartheta)$ is a variable-coefficient, narrow-stencil viscous derivative operator as defined by Mattssön [33], and the matrices $\left[\mu_{c}\right]$ and $\left[\kappa_{c}\right]$ are the artificial viscous coefficients and are related through the Prandtl number, $\operatorname{Pr}_{c}$. The fourth-order narrow-stencil viscous operator of Mattssön can be manipulated into a telescoping flux form. Thus, this type of artificial viscosity is consistent with the Lax Wendroff theorem. This is a critical part of this construction. If the chain rule was applied to the artificial viscosity terms, then Lax Wendroff would not immediately be satisfied regardless of what form is used for the convective terms.

The artificial viscosity $\mu_{c}$ is calculated based on a shock sensor [34],

$$
\begin{aligned}
\chi_{c}=\frac{b+|b|}{2|b|+c}, \quad b & =-\nabla \cdot v-\max \left(5 \sqrt{\omega \cdot \omega}, \frac{c}{20 \delta s}\right), \\
\omega & =\nabla \times v, \quad c=10^{-10},
\end{aligned}
$$

where $(\delta s)$ is the local grid spacing, and a smoothness function,

$$
\begin{gathered}
\chi_{s}=\frac{a-|a|}{2|a|+c}, \quad b=\left(\chi_{c}-1\right)|\mathcal{D}[\rho] \mathbf{s}-[\rho] \mathcal{D} \mathbf{s}-[s] \mathcal{D} \rho|, \\
a=\left(1-\chi_{c}\right)\left(b-\frac{M_{0}^{2}}{10^{3}}\right), \quad c=10^{-10},
\end{gathered}
$$

where $s$ is the thermodynamic entropy. The total sensor function is $\chi=\chi_{c}+\chi_{s}$. The formula for the artificial viscosity is

$$
\left(\mu_{c}\right)_{i}=\frac{\sqrt{\gamma p_{i} \rho_{i}} \delta s}{2}\left(\max \left(\chi_{i}, 0\right)-\min \left(\chi_{i} \delta s, 0\right)\right), \quad i=1,2, \ldots, N
$$

It is noted that this formula was constructed merely to show the shock properties of the different convective operators. For more intelligent artificial viscosity methods, see Refs. [35, 36, 37].

The above methodology was tested on Lax's shock tube problem. The initial conditions are described by

$$
(\rho, v, p)=\left(\begin{array}{ll}
(0.445,0.698,3.528) & \text { if } \quad x \leq 0 \\
(0.5,0,0.571) & \text { if } \quad x>0
\end{array},\right.
$$

where $x \in[-5,5]$. We use $\gamma=7 / 5$ and $\operatorname{Pr}_{c}=0.72$. Both the divergence and split forms of the convective terms were simulated with $N=200$ cells. The results are compared to a simulation of the divergence form simulated with $N=800$ cells in fig. 3. It is clear that the shock location is the same for both forms of the 


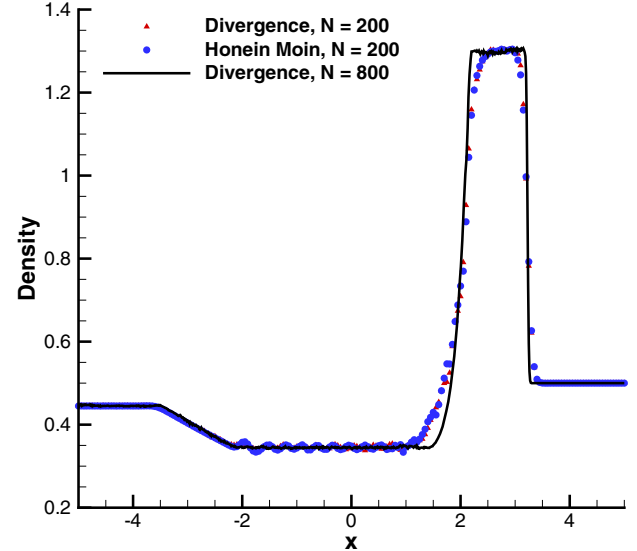

(a) Density

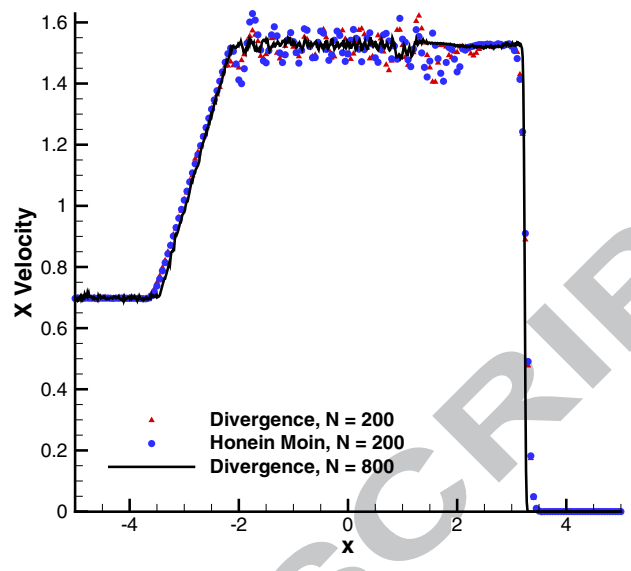

(b) Velcoity

Figure 3: Density and Velocity for Lax's shock tube problem comparing divergence and split forms are plotted.

convective terms. This result fully supports our proof. ${ }^{7}$

\section{Conclusion}

A class of split-form finite-difference operators are developed that satisfy the sufficient conditions of the Lax-Wendroff theorem. These operators are applicable to the conservation or divergence form of the conservation law, but facilitate operator splitting at the discrete level to improve accuracy or robustness, depending on the conservation law. The method for constructing a discretely telescoping split operator is illustrated for the (2-4-2) operator with a general splitting parameter. Higher order operators were also derived and are supplied in an accompanying text file. We also developed discretely telescoping operators for linear viscous terms.

The specific operators that are derived herein were tested on the conservation and canonical split forms of Burgers equation. Energy stability of the discrete form was proven, including boundary and interface closures. The split and conservation forms yield very similar results for Burgers equation. The solutions converged at least at the design order of accuracy in all cases, even when multiple domains were used with discontinuous grid resolutions across interfaces. The interface treatment that was tested herein have a negligible effect on the accuracy of the solution.

The new split-form operators are also tested on the Euler equations. It is demonstrated that the split form of Honein and Moin [12] can be cast into a telescoping flux form and that the shock location does not change for SBP discretizations of this alternative form.

\footnotetext{
${ }^{7}$ The split form operator adds significant additional cost, but greatly increases the flexibility of the method.
} 


\section{References}

[1] A. Arakawa, Computational design for long-term numerical integration of the equations of fluid motion: Two-dimensional incompressible flow. part i., Journal of Computational Physics 1 (1966) 119-143.

[2] W. J. Feiereisen, W. C. Reynolds, J. H. Ferziger, Numerical simulation of a compressible homogeneous, turbulent shear flow, Tech. Rep. TF-13, Stanford University (1981).

[3] A. Harten, On the symmetric form of systems of conservation laws with entropy, Journal of Computational Physics 49 (1983) 151-164.

[4] E. Tadmor, Skew-selfadjoint form of systems of conservation laws, Journal of Mathematical Analysis and Applications 103 (1984) 428-442.

[5] T. Zang, On the rotation and skew-symmetric forms for incompressible flow simulations, Applied Numerical Mathematics 7 (1991) 27-40.

[6] M. Gerritsen, P. Olsson, Designing an efficient solution strategy for fluid flows, Journal of Computational Physics 129 (1996) 245-262.

[7] G. A. Blaisdell, E. T. Spyropoulos, J. H. Qin, The effect of the formulation of nonlinear terms on aliasing errors in spectral methods, Applied Numerical Mathematics 21 (1996) 207-219.

[8] Y. Morinishi, T. S. Lund, O. V. Vasilyev, P. Moin, Fully conservative higher order finite difference schemes for incompressible flow, Journal of Computational Physics 143 (1998) 90-124.

[9] F. Ducros, F. Laporte, T. Soulères, V. Guinot, P. Moinat, B. Caruelle, High-order fluxes for conservative skew-symmetric-like schemes in structured meshes: Applications to compressible flows, Journal of Computational Physics 161 (2000) 114-139.

[10] H. C. Yee, M. Vinokur, M. J. Djomehri, Entropy splitting and numerical dissipation, Journal of Computational Physics 162 (2000) 33-81.

[11] H. Yee, B. Sjógreen, Designing adaptive low-dissipative high order schemes for long-time integrations, in: D. Drikakis, B. Geurts (Eds.), One: Turbulent Flow Computation, Kluwer Academic Publisher, 2001.

[12] A. E. Honein, P. Moin, Higher entropy conservation and numerical stability of compressible turbulence simulations, Journal of Computational Physics 201 (2004) 531-545.

[13] C. Kennedy, A. Gruber, Reducing aliasing formulations of the convective terms within the navier-stokes equations for a compressible fluid, Journal of Computational Physics 227(3) (2006) 1676-1700.

[14] P. K. Subbareddy, G. V. Candler, A fully discrete, kinetic energy consisten finite-volume scheme for compressible flows, Journal of Computational Physics 228(5) (2009) 1347-1364. 
[15] S. Pirozzoli, Generalized conservative approximations of split convective derivative operators, Journal of Computational Physics 229 (2010) 7180-7190.

[16] S. Pirozzoli, Stabilized non-dissipative approximations of euler equations in generalized curvilinear coordinates, Journal of Computational Physics 230 (2011) 2997-3014.

[17] S. Pirozzoli, Numerical methods for high-speed flows, Annual Review of Fluid Mechanics 43 (2011) 163-194.

[18] P. Lax, B. Wendroff, Systems of conservation laws, Communications on Pure and Applied Mathematics 13 (1960) 217-237.

[19] T. Y. Hou, P. G. L. Floch, Why nonconservative schemes converge to wrong solutions: error analysis, Mathematics of Computation 62 (206) (1994) 497-530.

[20] A. Jameson, The construction of discretely conservative finite volume schemes that also globally conserve energy or entropy, Journal of Scientific Computing 34 (2008) 152-187.

[21] B. Gustafsson, H.-O. Kreiss, Boundary conditions for time dependent problems with an artificial boundary, Journal of Computational Physics 30 (1979) 333-351.

[22] M. Svärd, J. Nordström, On the order of accuracy for difference approximations of initial-boundary value problems, Journal of Computational Physics 218 (2006) 333-352.

[23] M. H. Carpenter, J. Nordström, D. Gottlieb, A stable and conservative interface treatment of arbitrary spatial accuracy, Journal of Computational Physics 148 (1999) 341-365.

[24] N. K. Yamaleev, M. H. Carpenter, A systematic methodology for constructing high-order energy stable WENO schemes, Journal of Computational Physics 228 (2009) 4248-4272.

[25] T. C. Fisher, M. H. Carpenter, N. K. Yamaleev, S. H. Frankel, Boundary closures for fourth-order energy stable weighted essentially non-oscillatory finite difference schemes, Journal of Computational Physics 230 (2011) 3727-3752.

[26] M. H. Carpenter, J. Nordström, D. Gottlieb, Revisiting stable and conservative interface treatments of arbitrary spatial accuracy, Journal of Computational Physics 45(1-3) (2010) 118-150.

[27] J. Nordström, F. Ham, M. Shoeybi, E. van der Weide, M. Svärd, K. Mattsson, G. Iaccarino, J. Gong, A hybrid method for unsteady fluid flow, Computers and Fluids 38 (2009) 875-882.

[28] K. Mattsson, M. H. Carpenter, Stable and accurate interpolation operators for high-order multiblock finite-difference methods, SIAM J. on Scientific Computing 32 (2010) 2298.

[29] P. Olsson, J. Oliger, Energy and maximum norm estimates for nonlinear conservation laws, Tech. rep., Research Institute of Advanced Computer Science (1994). 
[30] M. H. Carpenter, D. Gottlieb, S. Abarbanel, Time-stable boundary conditions for finite-difference schemes solving hyperbolic systems: Methodology and application to high-order compact schemes, Journal of Computational Physics 111 (1994) 220-236.

[31] K. Mattsson, J. Nordström, Summation by parts operators for finite difference approximations of second derivatives, Journal of Computational Physics 199 (2004) 503-540.

[32] M. H. Carpenter, C. A. Kennedy, Fourth-order 2N-storage Runge-Kutta schemes, Tech. Rep. TM 109112, NASA (1994).

[33] K. Mattsson, Summation by parts operators for finite difference approximations of second-derivatives with variable coefficients, Journal of Scientific Computing 51 (2012) 650-682.

[34] I. Bermejo-Moreno, J. Larsson, S. K. Lele, Les of canonical shock-turbulence interaction, Tech. rep., Stanford Center for Turbulence Research Annual Research Briefs (2010).

[35] B. Fiorina, S. K. Lele, An artificial nonlinear diffusivity method for supersonic reacting flows with shocks, Journal of Computational Physics 222 (2007) 246-264.

[36] S. Kawai, S. K. Lele, Localized artificial diffusivity scheme for discontinuity capturing on curvilinear meshes, Journal of Computational Physics 227 (2008) 9498-9526.

[37] S. Kawai, S. K. Shankar, S. K. Lele, Assessment of localized artificial diffusivity scheme for large-eddy simulation of compressible turbulent flows, Journal of Computational Physics 229 (2010) 1739-1762.

[38] P. D. Lax, Hyperbolic Systems of Conservation Laws and the Mathematical Theory of Shock Waves, SIAM, Philadelphia, 1973.

\section{Appendix A. Discrete Conservation}

The summation-by-parts operators are discretely telescoping, as demonstrated in the following lemma.

Lemma Appendix A.1. All differentiation matrices that satisfy the SBP convention given in eq. (2.2) $\left(\mathcal{D}_{s b p}=\mathcal{P}^{-1} \mathcal{Q}\right)$ satisfy the column-sum condition $\mathbf{1}^{T} Q=(-1,0, \ldots, 0,1)$ where $\mathbf{1}^{T}=(1, \cdots, 1)$.

Proof. First-order consistency of $\mathcal{D}$ implies that $\mathcal{D}$ differentiates a constant exactly. Because $\mathcal{P}$ is nonsingular, the following matrix relations hold:

$$
\mathcal{P} \mathcal{D}_{s b p} \mathbf{1}=\mathcal{Q} \mathbf{1}=\mathbf{0}, \quad \mathbf{1}=(1, \cdots, 1)^{T}, \quad \mathbf{0}=(0, \cdots, 0)^{T}
$$

which demonstrates that all rows of $\mathcal{Q}$ sum to zero. Forming the dot-product of $\mathcal{Q}+\mathcal{Q}^{T}$ with the unit vector and combining the result with that obtained in eq. (A.1) yields

$$
\left(\mathcal{Q}+\mathcal{Q}^{T}\right) \mathbf{1}=\mathcal{Q}^{T} \mathbf{1}=\mathcal{B} \mathbf{1}=(-1,0, \ldots, 0,1)^{T}
$$


Taking the transpose of eq. (A.2) and combining it with the definition of an SBP operator yields

$$
\mathbf{1}^{T} \mathcal{Q}=\mathbf{1}^{T} \mathcal{P} \mathcal{D}_{s b p}=(-1,0, \ldots, 0,1)
$$

Thus, eq. (A.3) demonstrates that the columns of $\mathcal{D}_{s b p}$ are discretely telescoping in the $\mathcal{P}$ norm (i.e., all interior columns of the matrix $\mathcal{D}$ sum to zero, and the left and right boundary columns sum to -1 and 1 , respectively, after premultiplication by $\mathcal{P}$ ).

\section{Appendix B. Split Continuous Equations}

To utilize a split operator, the flux $f$ in eq. (2.1) must be the product of at least two functions: $f(u)=$ $v(u) w(u)$. Equation (2.1) is split by using the product rule

$$
u_{t}+\alpha f(u)_{x}+(1-\alpha)\left[v(u) w(u)_{x}+w(u) v(u)_{x}\right]=0
$$

This splitting does not change the weak solution to the governing equation, nor does it alter the Rankine Hugoniot relation. To show this, we start with the conservation law in one dimension:

$$
\frac{d}{d t} \int_{x_{L}}^{x_{R}} u d x+\left.f(u)\right|_{x_{L}} ^{x_{R}}=0, \quad x \in\left[x_{L}, x_{R}\right], \quad t \in[0, \infty)
$$

with a discontinuity located at $x_{d}$. At this point, note that neither eq. (2.1) nor eq. (B.1) is valid across the discontinuity. Both differential forms are valid on either side of the discontinuity, with the mild restriction that $w(u)_{x}$ and $v(u)_{x}$ exist in all regions where $f(u)_{x}$ exists. Equation (B.2) can be rewritten [38] as

$$
\frac{d}{d t} \int_{x_{L}}^{x_{d}^{-}} u d x+\frac{d}{d t} \int_{x_{d}^{+}}^{x_{R}} u d x+\left.f(u)\right|_{x_{L}} ^{x_{R}}=0
$$

where $u$ is smooth over the interval of integration. If we apply the Leibniz integral rule, then

$$
\int_{x_{L}}^{x_{d}^{-}} u_{t} d x+S u\left(x_{d}^{-}\right)+\int_{x_{d}^{+}}^{x_{R}} u_{t} d x-S u\left(x_{d}^{+}\right)+\left.f(u)\right|_{x_{L}} ^{x_{R}}=0
$$

where $S=\frac{d x_{d}}{d t}$ is the propagation speed of the discontinuity. Either eq. (2.1) or eq. (B.1) can be used to substitute for $u_{t}$, because the two forms are equal for smooth solutions. The conservation law becomes

$$
\int_{x_{L}}^{x_{d}^{-}}-f(u)_{x} d x+S u\left(x_{d}^{-}\right)+\int_{x_{d}^{+}}^{x_{R}}-f(u)_{x} d x-S u\left(x_{d}^{+}\right)+\left.f(u)\right|_{x_{L}} ^{x_{R}}=0
$$


Upon simplification, we get the Rankine Hugoniot relation

$$
\left[f\left(x_{d}^{+}\right)-f\left(x_{d}^{-}\right)\right]-S\left[u\left(x_{d}^{+}\right)-u\left(x_{d}^{-}\right)\right]=0
$$

Thus, both eq. (2.1) and eq. (B.1) satisfy the sufficient condition of the Lax-Wendroff theorem which specifies that the continuous weak solution of the discretized equations must satisfy the Rankine Hugoniot relations.

\section{Appendix C. High-Order Skew-Symmetric Fluxes}

\section{Appendix C.1. The Fourth-Order Operator: (3-6-3)}

The full (3-6-3) operator is available upon request. The use of electronic format necessitates the brevity of this presentation and easier implementation. For reference, the interior flux operator is

$$
f_{i}=\frac{1}{360}\left(\begin{array}{c}
u_{i-2} \\
u_{i-1} \\
u_{i} \\
u_{i+1} \\
u_{i+2} \\
u_{i+3}
\end{array}\right)^{T}\left(\begin{array}{cccccc}
2 & 0 & 0 & 1 & 0 & 0 \\
0 & -16 & 0 & -9 & 1 & 0 \\
0 & 0 & 74 & 45 & -9 & 1 \\
1 & -9 & 45 & 74 & 0 & 0 \\
0 & 1 & -9 & 0 & -16 & 0 \\
0 & 0 & 1 & 0 & 0 & 2
\end{array}\right)\left(\begin{array}{c}
u_{i-2} \\
u_{i-1} \\
u_{i} \\
u_{i+1} \\
u_{i+2} \\
u_{i+3}
\end{array}\right)
$$

Appendix C.2. The Complete Fifth-Order Operator: (4-8-4)

The full operator can be found in the accompanying text file. The flux for the interior is

$$
f_{i}=\frac{1}{840}\left(\begin{array}{c}
u_{i-3} \\
u_{i-2} \\
u_{i-1} \\
u_{i} \\
u_{i+1} \\
u_{i+2} \\
u_{i+3} \\
u_{i+4}
\end{array}\right)\left(\begin{array}{cccccccc}
-1 & 0 & 0 & 0 & -\frac{1}{2} & 0 & 0 & 0 \\
0 & \frac{29}{3} & 0 & 0 & \frac{16}{3} & -\frac{1}{2} & 0 & 0 \\
0 & 0 & -\frac{139}{3} & 0 & -28 & \frac{16}{3} & -\frac{1}{2} & 0 \\
0 & 0 & 0 & \frac{533}{3} & 112 & -28 & \frac{16}{3} & -\frac{1}{2} \\
-\frac{1}{2} & \frac{16}{3} & -28 & 112 & \frac{533}{3} & 0 & 0 & 0 \\
0 & -\frac{1}{2} & \frac{16}{3} & -28 & 0 & -\frac{139}{3} & 0 & 0 \\
0 & 0 & -\frac{1}{2} & \frac{16}{3} & 0 & 0 & \frac{29}{3} & 0 \\
0 & 0 & 0 & -\frac{1}{2} & 0 & 0 & 0 & -1
\end{array}\right)\left(\begin{array}{c}
u_{i-3} \\
u_{i-2} \\
u_{i-1} \\
u_{i} \\
u_{i+1} \\
u_{i+2} \\
u_{i+3} \\
u_{i+4}
\end{array}\right)
$$

\section{Appendix D. Conservative Finite-Difference Viscous Terms}

Many conservation laws of interest also have viscous terms. We show that for the linear viscous terms $\epsilon u_{x x}$ the summation-by-parts narrow-stencil approximation to the second derivative can be cast into a discretely telescoping form. The SBP finite-difference operators for the second derivative can be written as

$$
\mathcal{D}_{2}=\mathcal{D}^{2} \quad \text { or } \quad \mathcal{D}_{2}=\mathcal{P}^{-1}(\mathcal{R}+\mathcal{B S}), \quad \mathcal{R}=\mathcal{R}^{T}, \quad \mathbf{v}^{T} \mathcal{R} \mathbf{v} \leq 0
$$


where $\mathcal{S}$ represents a first derivative operator. In eq. D.1, $\mathcal{D}_{2}=\mathcal{D}^{2}$ is called the wide-stencil approximation to the second derivative, which requires that $\mathcal{S}=\mathcal{D} ; \mathcal{D}_{2}=\mathcal{P}^{-1}(\mathcal{R}+\mathcal{B S})$ is the narrow-stencil approximation to the second derivative and does not impose the same restriction on $\mathcal{S}$ [31]. The narrow-stencil approximation has superior accuracy and stability properties and should be used where possible.

The conservation form of the narrow stencil is found by constructing an approximation to the viscous flux, $\epsilon u_{x}$, at the flux points such that

$$
\Delta \overline{\mathbf{f}}_{V}=-\epsilon \Delta \overline{\mathcal{R}} \mathbf{u}=-\epsilon(\mathcal{R}+\mathcal{B S}) \mathbf{u}
$$

This is solved by using a linear system for an appropriate $\mathcal{R}$ and $\mathcal{B S}$ that satisfies the SBP condition. The viscous flux

$$
\overline{\mathbf{f}}_{V}=-\epsilon \overline{\mathcal{R}} \mathbf{u}
$$

yields a discretely telescoping approximation to $-\epsilon \mathcal{D}_{2} \mathbf{u}=\Delta \overline{\mathbf{f}}_{V}$. The operator $\overline{\mathcal{R}}$ for the $2 p=4$ scheme is presented herein, while the $\overline{\mathcal{R}}$ operators for $2 p=6$ and $2 p=8$ are available in the accompanying text file. For the fourth-order case, we begin with the existing fourth-order operator of Mattssön et al. [31], which can be expressed with the relation $\mathcal{D}_{2} \mathbf{u}=\Delta \overline{\mathcal{R}} \mathbf{u}=(\mathcal{R}+\mathcal{B S}) \mathbf{u}$, where

$$
\mathcal{R} \delta x=\left(\begin{array}{ccccccccccc}
-\frac{9}{8} & \frac{59}{48} & -\frac{1}{12} & -\frac{1}{48} & 0 & 0 & 0 & 0 & 0 & 0 & 0 \\
\frac{59}{48} & -\frac{59}{24} & \frac{59}{48} & 0 & 0 & 0 & 0 & 0 & 0 & 0 & 0 \\
-\frac{1}{12} & \frac{59}{48} & -\frac{55}{24} & \frac{59}{48} & -\frac{1}{12} & 0 & 0 & 0 & 0 & 0 & 0 \\
-\frac{1}{48} & 0 & \frac{59}{48} & -\frac{59}{24} & \frac{4}{3} & -\frac{1}{12} & 0 & 0 & 0 & 0 & 0 \\
0 & 0 & -\frac{1}{12} & \frac{4}{3} & -\frac{5}{2} & \frac{4}{3} & -\frac{1}{12} & 0 & 0 & 0 & 0 \\
0 & 0 & 0 & \ddots & \ddots & \ddots & \ddots & \ddots & 0 & 0 & 0 \\
0 & 0 & 0 & 0 & -\frac{1}{12} & \frac{4}{3} & -\frac{5}{2} & \frac{4}{3} & -\frac{1}{12} & 0 & 0 \\
0 & 0 & 0 & 0 & 0 & -\frac{1}{12} & \frac{4}{3} & -\frac{59}{24} & \frac{59}{48} & 0 & -\frac{1}{48} \\
0 & 0 & 0 & 0 & 0 & 0 & -\frac{1}{12} & \frac{59}{48} & -\frac{55}{24} & \frac{59}{48} & -\frac{1}{12} \\
0 & 0 & 0 & 0 & 0 & 0 & 0 & 0 & \frac{59}{48} & -\frac{59}{24} & \frac{59}{48} \\
0 & 0 & 0 & 0 & 0 & 0 & 0 & -\frac{1}{48} & -\frac{1}{12} & \frac{59}{48} & -\frac{9}{8}
\end{array}\right)
$$

and

$$
\mathcal{B S} \delta x=\left(\begin{array}{ccccccccccc}
\frac{11}{6} & -3 & \frac{3}{2} & -\frac{1}{3} & 0 & 0 & 0 & 0 & 0 & 0 & 0 \\
0 & 0 & 0 & 0 & 0 & 0 & 0 & 0 & 0 & 0 & 0 \\
\vdots & \vdots & \vdots & \vdots & \vdots & \vdots & \vdots & \vdots & \vdots & \vdots & \vdots \\
0 & 0 & 0 & 0 & 0 & 0 & 0 & 0 & 0 & 0 & 0 \\
0 & 0 & 0 & 0 & 0 & 0 & 0 & -\frac{1}{3} & \frac{3}{2} & -3 & \frac{11}{6}
\end{array}\right)
$$


Solving for $\overline{\mathcal{R}}$ in the relation $\Delta \overline{\mathcal{R}} \mathbf{u}=(\mathcal{R}+\mathcal{B S}) \mathbf{u}$ yields

$$
\overline{\mathcal{R}} \delta x=\left(\begin{array}{ccccccccc}
-\frac{11}{6} & 3 & -\frac{3}{2} & \frac{1}{3} & 0 & 0 & 0 & 0 & 0 \\
-\frac{9}{8} & \frac{59}{48} & -\frac{1}{12} & -\frac{1}{48} & 0 & 0 & 0 & 0 & 0 \\
\frac{5}{48} & -\frac{59}{48} & \frac{55}{48} & -\frac{1}{48} & 0 & 0 & 0 & 0 & 0 \\
\frac{1}{48} & 0 & -\frac{55}{48} & \frac{29}{24} & -\frac{1}{12} & 0 & 0 & 0 & 0 \\
0 & \frac{1}{12} & -\frac{5}{4} & \frac{5}{4} & -\frac{1}{12} & 0 & 0 & 0 & 0 \\
0 & 0 & \ddots & \ddots & \ddots & \ddots & 0 & 0 & 0 \\
0 & 0 & 0 & \frac{1}{12} & -\frac{5}{4} & \frac{5}{4} & -\frac{1}{12} & 0 & 0 \\
0 & 0 & 0 & 0 & \frac{1}{12} & -\frac{29}{24} & \frac{55}{48} & 0 & -\frac{1}{48} \\
0 & 0 & 0 & 0 & 0 & \frac{1}{48} & -\frac{55}{48} & \frac{59}{48} & -\frac{5}{48} \\
0 & 0 & 0 & 0 & 0 & \frac{1}{48} & \frac{1}{12} & -\frac{59}{48} & \frac{9}{8} \\
0 & 0 & 0 & 0 & 0 & -\frac{1}{3} & \frac{3}{2} & -3 & \frac{11}{6}
\end{array}\right) \text { (D.6) }
$$

Note that this operational form is only design order accurate for a constant diffusivity $\epsilon$.

A similar manipulation can be performed by using the sixth- and eighth-order diffusion operators. These operators are available in the accompanying text file. 